\title{
Fat content and fatty acid composition of forage fish and invertebrates in Prince William Sound, Alaska: factors contributing to among and within species variability
}

\author{
Sara J. Iverson ${ }^{1, *}$, Kathryn J. Frost ${ }^{2}$, Shelley L. C. Lang ${ }^{1}$ \\ ${ }^{1}$ Department of Biology, Dalhousie University, 1355 Oxford St., Halifax, Nova Scotia B3H 4J1, Canada \\ ${ }^{2}$ Alaska Department of Fish and Game, Division of Wildlife Conservation, 1300 College Road, Fairbanks, Alaska 99701, USA
}

\begin{abstract}
We determined the fat content and fatty acid composition of 26 species of fish and invertebrates $(n=1153)$ that are primary forage species of piscivorous seabirds and marine mammals in Prince William Sound (PWS), Alaska. Flatfish, shrimps and octopus had the lowest average fat contents $(\sim 1.0 \%)$, although some cods, as well as juvenile walleye pollock Theragra chalcogramma, Pacific herring Clupea harengus pallasi and pink salmon Oncorhynchus gorbuscha also ranged as low as 0.5 to $0.7 \%$ fat. The highest fat contents were found in eulachon Thaleichthys pacificus $(25 \%)$, adult herring (21\%) and the squid Berrytheuthis magister (5 to $13 \%$ ). Within species, fat content varied mostly with season, but also with size. Fatty acid signatures generally distinguished forage species, with up to $95 \%$ of individuals correctly classified using either discriminant or classification and regression tree (CART) analyses. Discriminant plots provided insight into the relationships between fatty acid signatures of different species. Species with similar life histories and diets clustered closer together, while those with the greatest differences in ecology differed most in their fatty acid patterns. Within some species, changes in fatty acid signatures were apparent with increasing size and were consistent with known dietary shifts reported from stomach contents analyses. Furthermore, fatty acid signatures of Age 0 (yr) pollock and herring in PWS were consistent with previous stomach contents analysis that indicated annual differences in the timing of dietary changes from eating zooplankton to piscivory. Overall, when size/age classes were taken into account, species classification using fatty acid signatures was improved. Our findings have important implications for evaluating diets and food web interactions of fish stocks, as well as at higher trophic levels. Despite individual variation within species, our results indicate that fatty acid signatures accurately characterize forage species in this ecosystem, and consequently can be used to study and perhaps estimate the species composition of diets of their predators.
\end{abstract}

KEY WORDS: Fatty acids $\cdot$ Forage fish $\cdot$ Food webs $\cdot$ Trophic interactions

\section{INTRODUCTION}

In Prince William Sound (PWS), one of the largest embayments $(90 \times 130 \mathrm{~km})$ in the Gulf of Alaska (GOA), many fish and invertebrate species, including

*E-mail: sara.iverson@dal.ca forage fishes such as capelin, herring, sandlance and juvenile pollock, represent critical resources for a number of apex predators including piscivorous fish, seabirds, pinnipeds and cetaceans (Springer \& Speckman 1997, Bowen \& Siniff 1999). In PWS and the GOA, a number of these apex predators as well as certain fish stocks have experienced declines over the past several decades (e.g. Frost \& Lowry 1994, Hayes \& Kuletz

(ㄷ) Inter-Research $2002 \cdot$ www.int-res.com 
1997, Merrick et al. 1997, Frost et al. 1999). Thus, it has become increasingly important to gain an understanding of the relationships between predator diets and the abundance and distribution of their prey in the ecosystem. The first step in this process must be to understand food webs and trophic interactions. Common methods used to examine diets of marine predators are based upon the recovery of extant prey remains in stomach contents and feces. While these methods can provide valuable information, for some taxa they may also involve serious biases in diet estimation which are well understood but difficult to avoid (e.g. Popova 1978, Jobling \& Breiby 1986, Pierce \& Boyle 1991). These biases include differential digestion and loss of both hard and soft parts, representation of only the last meal consumed, and the fact that stomachs are often empty and thus provide no information on diet. These factors have led to the development of alternative or complimentary methods to study trophic relationships.

One of these methods involves the use of fatty acids. Lipids, and especially fatty acids, have long been used as biological markers and general indicators of diet in marine ecology (Sargent et al. 1988). Fatty acids are the primary constituent of most lipids and in marine organisms they are most commonly composed of chains of 14 to 24 carbon atoms of varying degrees of unsaturation (i.e. containing one or more double bonds). These fatty acids generally remain intact through digestion, absorption and transport in the bloodstream, and are also taken up by tissues in their intact state. Thus, fatty acids can be deposited in animal tissue with minimal modification from diet and in a predictable way (Lee et al. 1971, Fraser et al. 1989, Xu et al. 1993, Graeve et al. 1994b, Iverson et al. 1995, 2001b, Kirsch et al. 1998, 2000). Additionally, animals can biosynthesize a relatively limited number of fatty acids (Cook 1985). These biochemical restrictions, coupled with the fact that fatty acids in the marine food web are exceptionally complex and diverse, provide the opportunity to use fatty acids to understand trophic interactions in marine ecosystems. Although a number of 'indicator' fatty acids exist which may be used as biological markers (e.g. Sargent et al. 1988, St. John \& Lund 1996), it is likely that the quantitative pattern of all fatty acids (i.e. the fatty acid signature, Iverson 1993) in a species or individual will be most informative at higher trophic levels (e.g. Iverson et al. 1999). It has been demonstrated that tissue fatty acids can be valuable in studying bottom-up trophic dynamics among and within fish and invertebrate species (e.g. Sargent et al. 1988, Fraser et al. 1989, Klungsøyr et al. 1989, Graeve et al. 1994a, Kharlamenko et al. 1995, St. John \& Lund 1996, Kirsch et al. 1998, Mayzaud et al. 1999). Additionally, once fatty acid patterns are characterized in prey or diet items, these patterns can be used to study the diets of higher trophic level predators (e.g. Klem 1935, Ackman \& Eaton 1966, Hooper et al. 1973, Rouvinen \& Kiiskinen 1989, Iverson 1993, Iverson et al. 1997a,b, 2001b, Raclot et al. 1998, Kirsch et al. 2000).

To use fatty acids to understand trophic interactions both among forage species and also near the top of the food web in PWS and other areas of the GOA, it is necessary to first characterize fatty acid patterns and their variation in the prey species assemblage. Thus, the aims of this study were to: (1) determine the fat content and fatty acid composition of the primary fish and invertebrate species that are known or potential prey of higher trophic level predators in PWS; (2) evaluate the degree to which these species can be reliably distinguished by their fatty acid signatures; and (3) where possible, evaluate the degree to which species signatures may vary ecologically with body size and over time and space.

\section{MATERIALS AND METHODS}

Sample collection. For the purpose of collections and analyses, PWS locations were divided into regions according to major fisheries zones as follows: central (C), northeast (NE), northwest (NW), southcentral (SC), southeast (SE) and southwest (SW) PWS. Forage species were collected throughout PWS in spring, summer and fall of 1994 through 1998. Collections were generally made using midwater trawls, seine and net sampling, during cruises associated with the APEX (Alaska Predator Ecosystem Experiment) project of the Exxon Valdez Oil Spill Restoration Program. Additional species and samples were collected in order to fill as many gaps in data as possible using various methods as opportunity provided. At collection, individuals or groups of individuals were sorted according to species and stored frozen in airtight plastic bags until analysis (within 6 mo or less). Although we would have preferred to obtain species over all representative ranges of size classes, seasons, locations and years, this was often not possible due to constraints associated with weather, collection and sampling. In many cases, cruises were designed to systematically sample specific areas in PWS in given years or to target certain species using species-specific selective gear.

In total, we analyzed the fat content and fatty acid composition of 1153 individual prey collected in PWS, representing 26 taxa. Of these, 163 individuals were analyzed as part of a previous preliminary study (Iverson et al. 1997b), but are included with the additional 990 currently analyzed individuals (Table 1). Species represented by a large size and/or age range, were further split into subgroups. Age classes (Year 0+, Year $1+$ and Year 2-adult) were estimated for individuals of 
Table 1. Morphometric and fat content data (mean \pm SEM) of prey species analyzed from Prince William Sound, Alaska $(\mathrm{n}=1148)$

\begin{tabular}{|c|c|c|c|c|c|c|c|}
\hline \multirow{2}{*}{$\begin{array}{l}\text { Family } \\
\text { Species }\end{array}$} & \multirow{2}{*}{$\begin{array}{l}\text { Age } / \text { size } \\
\text { class }\end{array}$} & \multirow[t]{2}{*}{ Season } & \multirow[t]{2}{*}{$\mathrm{n}$} & \multirow{2}{*}{$\begin{array}{l}\text { Length }(\mathrm{cm}) \\
\text { Mean } \pm \text { SEM }\end{array}$} & \multirow{2}{*}{$\begin{array}{c}\text { Mass }(\mathrm{g}) \\
\text { Mean } \pm \text { SEM }\end{array}$} & \multicolumn{2}{|c|}{ Fat content (\%) } \\
\hline & & & & & & Mean \pm SEM & Range \\
\hline \multicolumn{8}{|l|}{ Codfishes } \\
\hline Pacific cod & & Summer & 10 & $19.1 \pm 0.36$ & $51.7 \pm 2.95$ & $3.2 \pm 0.40$ & $1.1-5.3$ \\
\hline Gadus microcephalus & & Fall & 6 & $70.2 \pm 46.36$ & $130.7 \pm 17.09$ & $0.8 \pm 0.10$ & $0.6-1.3$ \\
\hline \multirow{7}{*}{$\begin{array}{l}\text { Walleye pollock } \\
\text { Theragra chalcogramma }\end{array}$} & $0+$ & Summer & 27 & $6.2 \pm 0.17$ & $1.8 \pm 0.20$ & $1.9 \pm 0.14$ & $0.7-3.5$ \\
\hline & & Fall & 26 & $11.7 \pm 0.47$ & $13.2 \pm 1.98$ & $2.0 \pm 0.27$ & $0.7-6.0$ \\
\hline & $1+$ & Spring & 17 & $13.4 \pm 0.22$ & $18.7 \pm 0.82$ & $5.1 \pm 0.45$ & $2.1-7.5$ \\
\hline & & Summer & 22 & $17.7 \pm 0.28$ & $38.4 \pm 1.64$ & $1.5 \pm 0.13$ & $0.6-2.8$ \\
\hline & & Fall & 64 & $18.9 \pm 0.24$ & $51.6 \pm 2.28$ & $2.3 \pm 0.13$ & $0.7-5.4$ \\
\hline & $2+$ & Spring & 36 & $21.9 \pm 0.75$ & $79.7 \pm 11.85$ & $1.9 \pm 0.09$ & $1.0-3.1$ \\
\hline & & Fall & 4 & $26.5 \pm 0.53$ & $136.3 \pm 10.82$ & $1.7 \pm 0.35$ & $1.0-2.3$ \\
\hline \multirow{3}{*}{$\begin{array}{l}\text { Pacific tomcod } \\
\text { Microgadus proximus }\end{array}$} & Small & Summer & 20 & $7.7 \pm 0.31$ & $3.4 \pm 0.55$ & $1.2 \pm 0.13$ & $0.4-2.2$ \\
\hline & Large & Summer & 23 & $21.1 \pm 0.58$ & $79.7 \pm 8.76$ & $1.6 \pm 0.13$ & $0.7-3.2$ \\
\hline & & Fall & 5 & $20.0 \pm 2.34$ & $77.8 \pm 34.43$ & $1.3 \pm 0.20$ & $0.7-1.8$ \\
\hline \multicolumn{8}{|l|}{ Greenlings } \\
\hline \multirow[t]{2}{*}{ Hexagrammos sp. } & & Summer & 6 & $27.9 \pm 2.09$ & $364.1 \pm 88.20$ & $4.4 \pm 0.43$ & $2.9-6.1$ \\
\hline & & Fall & 2 & $36.5 \pm 0.80$ & $573.4 \pm 8.11$ & $1.3 \pm 0.12$ & $1.2-1.5$ \\
\hline \multicolumn{8}{|l|}{ Herrings } \\
\hline \multirow{9}{*}{$\begin{array}{l}\text { Paciflc herring } \\
\text { Clupea harengus pallasi }\end{array}$} & $0+$ & Spring & 25 & $10.8 \pm 0.17$ & $9.3 \pm 0.45$ & $5.7 \pm 0.47$ & $1.2-9.9$ \\
\hline & & Summer & 171 & $10.3 \pm 0.19$ & $11.6 \pm 0.62$ & $4.3 \pm 0.23$ & $0.5-14.8$ \\
\hline & & Fall & 24 & $9.7 \pm 0.21$ & $7.2 \pm 0.65$ & $4.8 \pm 0.32$ & $1.1-7.2$ \\
\hline & $1+$ & Spring & 6 & $16.2 \pm 0.34$ & $35.4 \pm 1.98$ & $3.5 \pm 0.48$ & $1.9-4.6$ \\
\hline & & Summer & 23 & $15.7 \pm 0.30$ & $40.6 \pm 3.17$ & $10.4 \pm 1.01$ & $3.0-20.7$ \\
\hline & & Fall & 14 & $17.4 \pm 0.16$ & $50.8 \pm 3.05$ & $11.4 \pm 0.80$ & $6.2-16.0$ \\
\hline & 2-adult & Spring & 39 & $21.4 \pm 0.29$ & $98.5 \pm 5.43$ & $4.7 \pm 0.32$ & $2.0-9.6$ \\
\hline & & Summer & 21 & $20.9 \pm 0.40$ & $95.6 \pm 5.66$ & $8.0 \pm 0.86$ & $1.4-15.9$ \\
\hline & & Fall & 37 & $21.7 \pm 0.34$ & $119.9 \pm 6.07$ & $14.2 \pm 0.44$ & $6.7-20.4$ \\
\hline \multicolumn{8}{|l|}{ Righteye flounders } \\
\hline \multirow{2}{*}{\multicolumn{2}{|c|}{$\begin{array}{l}\text { Flathead sole } \\
\text { Hippoglossoides elassodon }\end{array}$}} & Summer & 22 & $15.9 \pm 0.82$ & $36.1 \pm 5.33$ & $1.9 \pm 0.20$ & $0.9-4.4$ \\
\hline & & Fall & 11 & $19.4 \pm 0.95$ & $63.5 \pm 12.05$ & $1.1 \pm 0.12$ & $0.7-1.8$ \\
\hline \multicolumn{2}{|l|}{$\begin{array}{l}\text { Pacific halibut } \\
\text { Hippoglossus stenolepis }\end{array}$} & Summer & 1 & 21.5 & 85.0 & 0.8 & - \\
\hline \multirow{3}{*}{\multicolumn{2}{|c|}{$\begin{array}{l}\text { Rex sole } \\
\text { Glyptocephalus zachirus }\end{array}$}} & Spring & 8 & $20.6 \pm 2.30$ & $56.7 \pm 26.14$ & $1.0 \pm 0.25$ & $0.1-2.4$ \\
\hline & & Summer & 11 & $17.1 \pm 0.58$ & $27.9 \pm 2.85$ & $1.2 \pm 0.12$ & $0.6-2.0$ \\
\hline & & Fall & 4 & $20.7 \pm 0.85$ & $46.0 \pm 7.15$ & $1.2 \pm 0.18$ & $0.8-1.7$ \\
\hline $\begin{array}{l}\text { Rock sole } \\
\text { Lepidopsetta bilineata }\end{array}$ & & Summer & 1 & 23.8 & 144.7 & 0.9 & - \\
\hline Yellowfin sole & & Summer & 9 & $22.0 \pm 0.51$ & $121.7 \pm 7.16$ & $1.5 \pm 0.09$ & $1.2-2.0$ \\
\hline Limanda aspera & & Fall & 8 & $28.6 \pm 0.89$ & $291.6 \pm 32.03$ & $4.7 \pm 0.92$ & $2.0-8.4$ \\
\hline Pleuronectidae & & Fall & 24 & $19.7 \pm 0.57$ & $61.8 \pm 6.78$ & $1.1 \pm 0.12$ & $0.4-2.1$ \\
\hline Rockfishes & & & & & & & \\
\hline Sebastes sp. & & Fall & 2 & $26.2 \pm 6.50$ & $471.4 \pm 297.55$ & $3.0 \pm 0.80$ & $2.2-3.8$ \\
\hline Sablefishes & & & & & & & \\
\hline $\begin{array}{l}\text { Blackcod } \\
\text { Anoplopoma fimbria }\end{array}$ & & Summer & 11 & $31.8 \pm 0.74$ & $336.2 \pm 23.02$ & $2.6 \pm 0.37$ & $0.5-4.6$ \\
\hline Salmonids & & & & & & & \\
\hline $\begin{array}{l}\text { Chum salmon } \\
\text { Oncorhynchus keta }\end{array}$ & & Summer & 7 & $10.2 \pm 0.68$ & $10.6 \pm 2.85$ & $1.4 \pm 0.09$ & $1.2-1.9$ \\
\hline Pink salmon & Smolt & Summer & 40 & $8.6 \pm 0.24$ & $6.2 \pm 0.53$ & $1.0 \pm 0.05$ & $0.6-2.1$ \\
\hline Oncorhynchus gorbuscha & Adult & Summer & 10 & $47.0 \pm 0.48$ & $1371.6 \pm 63.00$ & $3.8 \pm 0.36$ & $2.2-5.4$ \\
\hline Sandlances & & & & & & & \\
\hline Pacific sandlance & $0+$ & Summer & 66 & $9.0 \pm 0.17$ & $2.7 \pm 0.19$ & $3.5 \pm 0.17$ & $1.0-7.0$ \\
\hline Ammodytes hexapterus & $1+$ & Spring & 9 & $10.9 \pm 0.17$ & $9.1 \pm 0.45$ & $1.5 \pm 0.23$ & $0.8-2.4$ \\
\hline & & Summer & 32 & $13.0 \pm 0.14$ & $8.7 \pm 0.29$ & $5.2 \pm 0.25$ & $2.7-7.8$ \\
\hline Sculpins & & & & & & & \\
\hline Cottidae & & Summer & 6 & $20.6 \pm 1.78$ & $132.7 \pm 48.34$ & $1.5 \pm 0.35$ & $0.8-3.1$ \\
\hline & & Fall & 1 & 25.8 & 233.8 & 1.3 & - \\
\hline
\end{tabular}


Table 1 (continued)

\begin{tabular}{|c|c|c|c|c|c|c|c|}
\hline \multirow{2}{*}{$\begin{array}{l}\text { Family } \\
\text { Species }\end{array}$} & \multirow{2}{*}{$\begin{array}{l}\text { Age/size } \\
\text { class }\end{array}$} & \multirow[t]{2}{*}{ Season } & \multirow[t]{2}{*}{$\mathrm{n}$} & \multirow{2}{*}{$\begin{array}{l}\text { Length }(\mathrm{cm}) \\
\text { Mean } \pm \text { SEM }\end{array}$} & \multirow{2}{*}{$\begin{array}{c}\text { Mass }(\mathrm{g}) \\
\text { Mean } \pm \text { SEM }\end{array}$} & \multicolumn{2}{|c|}{ Fat content (\%) } \\
\hline & & & & & & Mean \pm SEM & Range \\
\hline \multicolumn{8}{|l|}{ Smelts } \\
\hline Capelin & & Spring & 19 & $12.4 \pm 0.19$ & $17.2 \pm 0.89$ & $3.2 \pm 0.31$ & $1.7-6.4$ \\
\hline Mallotus villosus & & Summer & 53 & $13.1 \pm 0.08$ & $14.6 \pm 0.58$ & $1.4 \pm 0.14$ & $0.6-5.8$ \\
\hline $\begin{array}{l}\text { Eulachon } \\
\text { Thaleichthys pacificus }\end{array}$ & & Spring & 20 & $15.1 \pm 0.28$ & $18.4 \pm 1.49$ & $19.0 \pm 0.52$ & $15.0-25.3$ \\
\hline $\begin{array}{l}\text { Rainbow smelt } \\
\text { Osmerus mordax }\end{array}$ & & na & 4 & $20.5 \pm 0.55$ & $73.4 \pm 12.30$ & $3.6 \pm 0.87$ & $2.4-6.1$ \\
\hline \multicolumn{8}{|l|}{ Shrimp } \\
\hline Pandalus borealis & & $\begin{array}{c}\text { Spring } \\
\text { Summer } \\
\text { na }\end{array}$ & $\begin{array}{l}6 \\
2 \\
7\end{array}$ & $\begin{array}{l}8.7 \pm 0.35 \\
7.9 \pm 0.33 \\
8.4 \pm 0.14\end{array}$ & $\begin{array}{l}3.2 \pm 0.36 \\
2.0 \pm 0.48 \\
2.7 \pm 0.08\end{array}$ & $\begin{array}{l}0.9 \pm 0.09 \\
1.4 \pm 0.01 \\
1.7 \pm 0.09\end{array}$ & $\begin{array}{l}0.7-1.3 \\
1.3-1.4 \\
1.4-2.1\end{array}$ \\
\hline Pandalus sp. & & Fall & $2^{*}$ & na & $1.6 \pm 0.04$ & $1.1 \pm 0.11$ & $1.0-1.2$ \\
\hline \multicolumn{8}{|l|}{ Squid } \\
\hline Magister armhook & Small & Fall & 8 & na & $8.4 \pm 1.65$ & $8.0 \pm 0.96$ & $4.9-13.2$ \\
\hline Berrytheuthis magister & $\begin{array}{l}\text { Medium } \\
\text { Large }\end{array}$ & $\begin{array}{l}\text { Fall } \\
\text { Fall }\end{array}$ & $\begin{array}{l}2 \\
2\end{array}$ & $\begin{array}{l}\text { na } \\
\text { na }\end{array}$ & $\begin{aligned} 80.8 & \pm 0.33 \\
784.9 & \pm 26.25\end{aligned}$ & $\begin{array}{l}3.5 \pm 0.58 \\
4.8 \pm 0.19\end{array}$ & $\begin{array}{l}2.9-4.1 \\
4.6-5.0\end{array}$ \\
\hline $\begin{array}{l}\text { North Pacific bobtail } \\
\text { Rossia pacifica }\end{array}$ & & Fall & 22 & na & $15.0 \pm 1.87$ & $2.2 \pm 0.09$ & $1.2-3.1$ \\
\hline \multirow{3}{*}{\multicolumn{2}{|c|}{ Gonatidae }} & Spring & 28 & $18.7 \pm 0.71$ & $25.9 \pm 3.25$ & $1.6 \pm 0.07$ & $0.9-2.3$ \\
\hline & & Summer & 47 & $26.5 \pm 0.93$ & $37.8 \pm 3.37$ & $2.2 \pm 0.15$ & $1.0-4.8$ \\
\hline & & Fall & 8 & $49.2 \pm 5.14$ & $148.7 \pm 35.74$ & $3.0 \pm 0.46$ & $1.1-4.6$ \\
\hline \multicolumn{8}{|l|}{ Octopus } \\
\hline \multirow[t]{3}{*}{ Octopus sp. } & & Summer & 2 & $36.5 \pm 3.50$ & $303.2 \pm 106.75$ & $1.6 \pm 0.28$ & $1.3-1.9$ \\
\hline & & Fall & 3 & $52.7 \pm 15.05$ & $1081.1 \pm 548.05$ & $1.1 \pm 0.15$ & $0.9-1.4$ \\
\hline & & na & 2 & $38.6 \pm 1.85$ & $252.8 \pm 93.78$ & $1.1 \pm 0.03$ & $1.1-1.1$ \\
\hline \multicolumn{8}{|c|}{$\begin{array}{l}\text { All values were derived from whole prey that were ground and analyzed individually. In cases where prey were too small t } \\
\text { be analyzed separately, several individuals were combined for analysis and considered to be an } \mathrm{n} \text { of } 1 \text {. Five samples ( } 4 \text { pollock } \\
1 \text { herring) were excluded from this table as they did not have associated size data to allow age classification }\end{array}$} \\
\hline $\begin{array}{l}{ }^{*} \text { Each sample consisted of } \\
\text { na: not available }\end{array}$ & 23 individu & l shrimp gr & d toge & & & & \\
\hline
\end{tabular}

Pacific herring, walleye pollock and Pacific sandlance using size at age data for these species (Incze et al. 1988, Sturdevant 1996, Blackburn \& Anderson 1997, Ciannelli 1997). For other species represented by a large size range (e.g. Pacific tomcod and armhook squid), individuals were grouped in size classes according to the length or mass distributions represented (size and mass measurements described below). Five individuals (4 pollock, 1 herring) did not have associated size data to allow age classification and thus, were not included in any reports or analyses that used age classes.

Fat content and fatty acid analysis. After recording length (nose to fork of tail $\pm 0.1 \mathrm{~cm}$ ) and mass $( \pm 0.1 \mathrm{~g}$ ) of each whole prey, each was ground individually and lipids were quantitatively extracted in duplicate aliquots; fat content was expressed as an average (of total fresh mass) of the 2 duplicates. In cases where prey were too small to analyze separately, several individuals were combined for analyses; in these cases each group analysis was considered to be equal to a sample size of one $(n=1)$. Although it may have increased within-species variability, we did not attempt to remove gut contents of any prey prior to analysis, as our aim was to characterize them as prey, which are eaten whole by marine predators.

At the onset of this project, prey samples were extracted and analyzed using the Bligh \& Dyer (1959) method. This method and that of Folch et al. (1957) are well recognized for total extraction of lipids from animal tissue (Ackman 1980, Christie 1982). The primary advantage of the Bligh \& Dyer method is a reduction in the solvent:sample ratio and, since its development over 30 yr ago, it has become a standard method for the determination of total lipids, especially by fisheries laboratories (e.g. Bailey \& Wells 1994, Payne et al. 1999, Smedes \& Askland 1999). However, in the course of our study, we discovered that samples of a known very high fat content were significantly underestimated using the Bligh \& Dyer method but not by the Folch method, although the fatty acid composition remained identical under both. A highly significant linear relationship was demonstrated between the 2 methods, which permits the correction of reported lipid 
levels in samples previously analyzed using Bligh \& Dyer (Iverson et al. 2001a). Thus, all samples that had been analyzed using the Bligh \& Dyer method in this study were either re-analyzed using the Folch method or corrected using the derived equation. All prey samples collected since 1996 were analyzed using the Folch method.

Fatty acid methyl esters were prepared directly from $100 \mathrm{mg}$ of the pure extracted lipid according to Iverson et al. (1997b). Duplicate analyses of fatty acid methyl esters were performed on samples using temperature-programmed gas liquid chromatography according to Iverson et al. (1997b), on a Perkin Elmer Autosystem II Capillary FID gas chromatograph (GC) fitted with a $30 \mathrm{~m} \times 0.25 \mathrm{~mm}$ (in diameter) column coated with $50 \%$ cyanopropyl polysiloxane $(0.25 \mu \mathrm{m}$ film thickness; J\&W DB-23) and linked to a computerized integration system (Turbochrom 4.1 software, PE Nelson). Identification of fatty acids and isomers were determined from a number of sources including known standard mixtures (Nu Check Prep), silver-nitrate (argentation) chromatography and gas chromatography (GC) mass spectrometry (HewlettPackard 6890 Gas Chromatograph, 1:20 split injection, Micromass Autospec oa-TOF mass spectrometer, operated at 1000 resolution, scanning masses 120 to 450). Individual fatty acids are expressed as weight percent of total fatty acids after employing mass response factors relative to 18:0. Theoretical relative response factors were used for this purpose (Ackman 1991), with minor adjustments made after tests with accurate quantitative standard mixtures ( $\mathrm{Nu}$ Check Prep). GC columns were kept in good condition throughout the study by changing septa daily, cleaning the injector liner regularly, by use of a guard column, and by frequent replacement. All sample chromatograms and fatty acid identifications were individually checked and corrected and reintegrated if necessary. Fatty acids are expressed as weight percent of total fatty acids and are designated by shorthand nomenclature of carbon chain length:number of double bonds and location (n-x) of the double bond nearest the terminal methyl group. All means are presented as mean \pm SEM throughout, unless otherwise indicated.

Statistical analyses. Due to the constraints of sample collection, some species were represented by small sample sizes. However, other species were represented by sufficient numbers to allow statistical testing of species differences as well as some evaluation of variability with size or age class, year, season or location.

Species differences were evaluated using a combination of univariate and multivariate techniques. Discriminant analysis (SPSS) was performed using the 17 fatty acids which had the largest overall variance and an overall mean of $\geq 0.4 \%$ of total fatty acids. The exceptions were 22:5n-3 and 24:1. Despite its large overall variance, $22: 5 n-3$ was not used as it may be an intermediate between 20:5n-3 and 22:6n-3 (e.g. Ackman et al. 1988), and thus, is less useful as a dietary indicator at higher trophic levels. As a result of minor shifts in the stationary phase of our columns (at factory production), the ability to identify all isomers of $24: 1$ was not consistent for all samples during the course of the study and it was therefore not used in statistical analyses. Percentage values for fatty acids were transformed into log ratios prior to discriminant analysis by first renormalizing the values for the 17 fatty acids over $100 \%$ and then dividing the value for each of the other fatty acids by the value for 18:0. The resulting 16 ratios were then log transformed and used in the analyses. Since the log of 0 cannot be taken, 0 values for any of the 17 fatty acids were changed to $0.005 \%$ prior to the calculation of the log ratio $(\mathrm{n}=3$ for $16: 4 \mathrm{n}-1, \mathrm{n}=11$ for $22: 1 \mathrm{n}-11$ and $n=2$ for $22: 1 \mathrm{n}-9$ ). This value of $0.005 \%$ was selected as a value that is below what we considered to be the minimum detectable level $(0.01 \%)$ but which would not be so small as to result in extreme outliers following transformation. Wilk's $\lambda$ was used to indicate the power of the discriminant analysis to separate groups (smaller values indicating greater success). The percent of cases correctly classified were used to evaluate the performance of the classification function and the classifications were cross validated using a jackknife procedure (leave-one-out cross-validation, SPSS). The predicted group membership of individuals based on the classification function was examined to determine into which group individuals were misclassified. Since the n of any group used in discriminant analysis must be greater than the number of variables used (Stevens 1986, Legendre \& Legendre 1998), only groups with 17 or more samples were included in these analyses.

Species differences in fatty acid composition were also compared using classification and regression tree (CART) analysis (S-Plus, Statistical Sciences) according to Smith et al (1997) and Iverson et al (1997a). Briefly, CART is a non-parametric technique for classifying data which proceeds by recursively partitioning data by sequentially selecting the 'best' variable (fatty acid) which separates the data into 2 groups (nodes) that are as different as possible (Clark \& Pregibon 1992). The splitting continues in a tree-like form until 1 of 2 stopping criteria (based on minimum deviance of a final node or minimum number of observations in that node) is met and a classification is made along with the associated misclassification or error rate. One of the advantages of CART is that there are no restrictions on the number of variables that can be used in the analysis; therefore, all fatty acids with the exception of $24: 1$ 
were used in these analyses. A restriction on CART is that groups less than $\mathrm{n}=5$ cannot be classified; therefore, species with sample sizes of 4 or less were excluded from these analyses. Classification results obtained using CART were compared to those obtained using discriminant analysis.

\section{RESULTS}

\section{Fat content variation within and among species}

The fat content of most species averaged $3 \%$ or less (Table 1). Flatfishes, shrimps and octopus had the lowest average fat contents $(\sim 1.0 \%)$ of the species analyzed. However, there was considerable within-species variability, with some cods, juvenile pollock and herring (Age 0+), and pink salmon smolts ranging even lower at 0.5 to $0.7 \%$ fat. The highest fat contents were found in eulachon (up to $25 \%$ ) and in Age 1+ and 2adult herring (up to $21 \%$ ). The next highest fat contents (5 to $13 \%$ ) were found in small Berrytheuthis magister. Individuals of all other species were $<10 \%$ fat. Within species, fat content appeared to vary mostly with season, but also with size. The unequal distribution of samples across size classes, season and year precluded rigorous analysis of the effects of these factors on fat content in most species. However, across all years, the fat content of herring was highest in 1+ and 2-adult age classes, and in fall compared to other seasons ( $\mathrm{p}<0.001,2$-way ANOVA, $\mathrm{n}=360$ ). In contrast, pollock was higher in fat in the spring $(\mathrm{p}<0.001)$ and in $1+$ compared to either $0+$ or $2+$ age individuals $(\mathrm{p}=$ 0.005, $\mathrm{n}=196)$. During summer, Age 1+ sandlance were higher in fat than $0+$ samples $(\mathrm{p}<0.001, \mathrm{n}=98)$. Fat content was higher in Pacific tomcod in summer compared to fall ( $\mathrm{p}<0.001, \mathrm{n}=16$ ), and in capelin in the spring compared to summer $(\mathrm{p}<0.001, \mathrm{n}=72$, Table 1).

\section{Fatty acid patterns across species}

Approximately 66 fatty acids were routinely identified and quantified in all species. Given this large number, we present summary data for only those fatty acids that averaged $\geq 0.2 \%$ of total fatty acids among all species analyzed (Table 2). (Note: complete data, including all fatty acids quantified by individual, with size classes and years, locations and seasons of collection, are available upon request from the corresponding author.) The most abundant fatty acids generally included $14: 0,16: 0,16: 1 n-7,16: 4 n-1,18: 0,18: 1 n-9$, $18: 1 n-7,18: 2 n-6,18: 3 n-3,18: 4 n-3,20: 1 n-11,20: 1 n-9$, 20:4n-6, 20:5n-3, 22:1n-11, 22:1n-9, 22:5n-3 and 22:6n-3, which together accounted for about $90 \%$ of total fatty acids. Although the most abundant fatty acids were generally similar across species, the levels of these fatty acids varied greatly among species. For instance, 14:0 was lowest in Pacific cod and tomcod (0.4 to $1.0 \%)$ and highest in herring $(14.0 \%)$ and eulachon $(9.5 \%)$. The lowest levels of $16: 1 \mathrm{n}-7$ were consistently found in squids and octopus (0.8 to $1.1 \%$ ), while the highest levels were found in yellowfin sole, flathead sole and rex sole (12 to $16 \%) ; 18: 1 n-9$ was as low as 1 to $2 \%$ in squids, octopus and pollock and as high as $40 \%$ in eulachon. Both 18:2n-6 and 18:3n-3 were lowest in rex sole, pollock and Pacific cod (0.02 to $0.3 \%)$ and highest in greenlings (up to $6 \%$ each). The lowest levels of $20: 1 \mathrm{n}-11$ and $22: 1 \mathrm{n}-11$ (0 to $0.06 \%$ ) were found in pink salmon smolts, and Age 0+ herring and sandlance, while the highest levels (15 to $22 \%$ ) were found in Age 2+ herring followed by capelin. Similar to the trends for $20: 1 \mathrm{n}-11$ and $22: 1 \mathrm{n}-11$, the lowest levels of $20: 1 n-9$ were also found in pink salmon smolts, and Age 0+ herring and sandlance. However, the highest level ( $7 \%$ ) of 20:1n-9 was found in Rossia pacifica (bobtail squid). Fatty acids 20:5n-3 and $22: 6 n-3$ were both lowest in eulachon (1 to $4 \%$ ). Levels of 20:5n-3 were highest in tomcod, squid and shrimps (20 to $21 \%$ ), while $22: 6 n-3$ was highest in tomcod, pink salmon smolts and Pacific cod (38 to $43 \%$ ) (Table 2).

Although species characteristics were evident, there was also significant within-species variation. Given the large number of fatty acids identified, multivariate assessments provide clearer indications of species differences and patterns. Despite within-species variation, prey were generally readily and accurately distinguished from one another based upon their overall fatty acid signature. Discriminant analysis was performed on 13 species (with $n \geq 17$ ) using 17 of the 18 abundant fatty acids listed above, excluding 22:5n-3, which together accounted for about $88 \%$ of total fatty acids. This analysis separated species with $91.0 \%$ (955 of 1050) of individuals correctly classified and the group centroids for the first and second discriminant functions illustrate some differences between species (Fig. 1). For instance, eulachon were clearly well separated from all other species on the first 2 discriminant functions and were also $100 \%$ correctly classified overall (i.e. 20/20, Table 3). In contrast, the 4 groups of flatfish (righteye flounders) were closely associated with one another on the first 2 discriminant functions (Fig. 1) and when misclassified overall, were always misclassified as another flatfish (Table 3). If all flatfish were grouped together, the correct separation of all groups increased from 91.0 to $93.0 \%$, and $100 \%$ of flatfish were correctly classified. Capelin and squid were also classified well (97.2 to $100 \%)$. In contrast, 
Table 2. Fatty acid composition (mass \%) of prey species from Prince William Sound, Alaska (n=1148). Values are means \pm SEM of fatty acids (36 out of 66 ) which averaged $\geq 0.2 \%$ among all prey analyzed. ${ }^{*}$ Designates the 17 fatty acids used in discriminant analyses. Complete data, including all fatty acids quantified among individuals with size classes, years, locations and seasons are available on request from the corresponding author. SAT = saturated, MUFA $=$ monounsaturated, PUFA $=$ polyunsaturated

\begin{tabular}{|c|c|c|c|c|c|c|}
\hline \multirow{2}{*}{\multicolumn{2}{|c|}{$\begin{array}{l}\text { Pacific cod } \\
\text { Age/size class: }\end{array}$}} & \multicolumn{3}{|c|}{$\begin{array}{l}\text { Codfishes } \\
\text {. }\end{array}$} & \multicolumn{2}{|c|}{$\longrightarrow$ Pacific tomcod } \\
\hline & & \multirow{2}{*}{$\begin{array}{l}0+ \\
53\end{array}$} & \multirow{2}{*}{$\begin{array}{c}\text { Walleye pollock - } \\
1+ \\
103\end{array}$} & \multirow{2}{*}{$\begin{array}{l}2+ \\
40\end{array}$} & \multirow{2}{*}{$\begin{array}{c}\text { Small } \\
20\end{array}$} & \multirow{2}{*}{$\begin{array}{l}\text { Large } \\
28\end{array}$} \\
\hline n: & 16 & & & & & \\
\hline \multicolumn{7}{|l|}{ SAT } \\
\hline $14: 0^{*}$ & $1.58 \pm 0.25$ & $2.73 \pm 0.17$ & $4.07 \pm 0.15$ & $3.88 \pm 0.18$ & $2.28 \pm 0.23$ & $1.56 \pm 0.09$ \\
\hline $15: 0$ & $0.27 \pm 0.01$ & $0.31 \pm 0.01$ & $0.26 \pm 0.01$ & $0.24 \pm 0.01$ & $0.35 \pm 0.02$ & $0.44 \pm 0.03$ \\
\hline iso $16: 0$ & $0.26 \pm 0.02$ & $0.23 \pm 0.02$ & $0.12 \pm 0.01$ & $0.17 \pm 0.01$ & $0.31 \pm 0.03$ & $0.30 \pm 0.02$ \\
\hline $16: 0^{*}$ & $14.82 \pm 0.30$ & $17.74 \pm 0.36$ & $14.81 \pm 0.28$ & $16.07 \pm 0.30$ & $18.02 \pm 0.35$ & $15.34 \pm 0.16$ \\
\hline $17: 0$ & $0.27 \pm 0.03$ & $0.20 \pm 0.02$ & $0.29 \pm 0.03$ & $0.44 \pm 0.07$ & $0.39 \pm 0.03$ & $0.51 \pm 0.04$ \\
\hline $18: 0^{*}$ & $4.49 \pm 0.23$ & $4.01 \pm 0.13$ & $3.02 \pm 0.10$ & $2.93 \pm 0.14$ & $4.49 \pm 0.13$ & $4.82 \pm 0.12$ \\
\hline \multicolumn{7}{|l|}{ MUFA } \\
\hline $14: 1 n-9$ & $0.08 \pm 0.02$ & $0.22 \pm 0.02$ & $0.17 \pm 0.01$ & $0.23 \pm 0.02$ & $0.27 \pm 0.03$ & $0.09 \pm 0.02$ \\
\hline $16: 1 n-11$ & $0.41 \pm 0.02$ & $0.45 \pm 0.02$ & $0.36 \pm 0.01$ & $0.29 \pm 0.01$ & $0.53 \pm 0.02$ & $0.47 \pm 0.03$ \\
\hline $16: 1 n-9$ & $0.38 \pm 0.02$ & $0.23 \pm 0.01$ & $0.16 \pm 0.00$ & $0.15 \pm 0.01$ & $0.30 \pm 0.02$ & $0.44 \pm 0.02$ \\
\hline $16: 1 n-7^{*}$ & $2.92 \pm 0.34$ & $3.39 \pm 0.25$ & $5.41 \pm 0.18$ & $6.16 \pm 0.27$ & $2.83 \pm 0.26$ & $4.81 \pm 0.35$ \\
\hline $17: 1$ & $0.28 \pm 0.02$ & $0.16 \pm 0.02$ & $0.11 \pm 0.01$ & $0.15 \pm 0.01$ & $0.09 \pm 0.02$ & $0.30 \pm 0.04$ \\
\hline 18:1n-11 & $0.75 \pm 0.15$ & $0.60 \pm 0.08$ & $1.13 \pm 0.07$ & $0.69 \pm 0.06$ & $0.34 \pm 0.05$ & $0.40 \pm 0.13$ \\
\hline $18: 1 n-9^{*}$ & $13.62 \pm 0.80$ & $9.19 \pm 0.26$ & $8.73 \pm 0.35$ & $10.31 \pm 0.40$ & $8.86 \pm 0.32$ & $8.54 \pm 0.38$ \\
\hline $18: 1 \mathrm{n}-7^{*}$ & $3.92 \pm 0.14$ & $2.97 \pm 0.17$ & $3.38 \pm 0.15$ & $4.21 \pm 0.29$ & $2.51 \pm 0.06$ & $5.64 \pm 0.24$ \\
\hline $18: 1 n-5$ & $0.40 \pm 0.02$ & $0.57 \pm 0.04$ & $0.50 \pm 0.01$ & $0.39 \pm 0.01$ & $0.62 \pm 0.05$ & $0.35 \pm 0.02$ \\
\hline $20: 1 \mathrm{n}-11^{*}$ & $1.73 \pm 0.33$ & $1.92 \pm 0.42$ & $6.45 \pm 0.40$ & $4.71 \pm 0.37$ & $0.36 \pm 0.04$ & $0.87 \pm 0.07$ \\
\hline $20: 1 \mathrm{n}-9^{*}$ & $1.68 \pm 0.19$ & $1.60 \pm 0.10$ & $2.20 \pm 0.06$ & $1.84 \pm 0.09$ & $1.76 \pm 0.26$ & $0.86 \pm 0.07$ \\
\hline $20: 1 n-7$ & $0.42 \pm 0.07$ & $0.15 \pm 0.01$ & $0.21 \pm 0.00$ & $0.23 \pm 0.01$ & $0.18 \pm 0.04$ & $1.05 \pm 0.10$ \\
\hline $22: 1 \mathrm{n}-11^{*}$ & $0.92 \pm 0.21$ & $2.02 \pm 0.38$ & $6.47 \pm 0.42$ & $4.98 \pm 0.47$ & $0.67 \pm 0.14$ & $0.24 \pm 0.03$ \\
\hline $22: 1 \mathrm{n}-9^{*}$ & $0.23 \pm 0.03$ & $0.42 \pm 0.04$ & $0.61 \pm 0.02$ & $0.92 \pm 0.10$ & $0.16 \pm 0.02$ & $0.10 \pm 0.01$ \\
\hline \multicolumn{7}{|l|}{ PUFA } \\
\hline $16: 2 n-4$ & $0.57 \pm 0.04$ & $0.41 \pm 0.03$ & $0.44 \pm 0.03$ & $0.26 \pm 0.03$ & $0.66 \pm 0.06$ & $0.41 \pm 0.03$ \\
\hline $16: 3 n-6$ & $0.13 \pm 0.03$ & $0.30 \pm 0.04$ & $0.26 \pm 0.03$ & $0.51 \pm 0.03$ & $0.15 \pm 0.02$ & $0.16 \pm 0.03$ \\
\hline $16: 3 n-4$ & $0.08 \pm 0.03$ & $0.37 \pm 0.05$ & $0.31 \pm 0.04$ & $0.36 \pm 0.03$ & $0.20 \pm 0.01$ & $0.26 \pm 0.04$ \\
\hline $16: 4 n-1^{*}$ & $0.28 \pm 0.05$ & $0.36 \pm 0.04$ & $0.56 \pm 0.07$ & $0.29 \pm 0.03$ & $0.26 \pm 0.02$ & $0.24 \pm 0.05$ \\
\hline $18: 2 n-6^{*}$ & $0.60 \pm 0.05$ & $0.82 \pm 0.04$ & $0.70 \pm 0.02$ & $0.57 \pm 0.02$ & $1.02 \pm 0.05$ & $1.11 \pm 0.13$ \\
\hline $18: 3 n-3^{*}$ & $0.34 \pm 0.05$ & $0.59 \pm 0.03$ & $0.55 \pm 0.02$ & $0.29 \pm 0.03$ & $0.68 \pm 0.03$ & $0.68 \pm 0.09$ \\
\hline $18: 4 n-3^{*}$ & $0.41 \pm 0.08$ & $1.54 \pm 0.09$ & $1.65 \pm 0.07$ & $0.86 \pm 0.07$ & $1.21 \pm 0.07$ & $0.72 \pm 0.08$ \\
\hline $20: 2 n-6$ & $0.31 \pm 0.02$ & $0.24 \pm 0.01$ & $0.19 \pm 0.01$ & $0.18 \pm 0.01$ & $0.35 \pm 0.02$ & $0.63 \pm 0.06$ \\
\hline $20: 4 n-6^{*}$ & $2.67 \pm 0.22$ & $1.04 \pm 0.07$ & $0.75 \pm 0.04$ & $0.91 \pm 0.05$ & $1.33 \pm 0.18$ & $3.30 \pm 0.14$ \\
\hline $20: 4 n-3$ & $0.48 \pm 0.05$ & $0.62 \pm 0.02$ & $0.66 \pm 0.02$ & $0.55 \pm 0.03$ & $0.60 \pm 0.02$ & $0.58 \pm 0.05$ \\
\hline $20: 5 n-3^{*}$ & $10.97 \pm 0.53$ & $13.69 \pm 0.32$ & $11.72 \pm 0.22$ & $13.04 \pm 0.47$ & $13.21 \pm 0.27$ & $16.55 \pm 0.49$ \\
\hline $21: 5 n-3$ & $0.22 \pm 0.03$ & $0.32 \pm 0.02$ & $0.34 \pm 0.01$ & $0.40 \pm 0.02$ & $0.25 \pm 0.01$ & $0.32 \pm 0.02$ \\
\hline $22: 5 n-6$ & $0.33 \pm 0.04$ & $0.26 \pm 0.01$ & $0.18 \pm 0.01$ & $0.18 \pm 0.01$ & $0.27 \pm 0.01$ & $0.41 \pm 0.03$ \\
\hline $22: 5 n-3$ & $2.31 \pm 0.19$ & $1.04 \pm 0.04$ & $1.11 \pm 0.02$ & $1.30 \pm 0.04$ & $1.06 \pm 0.09$ & $4.15 \pm 0.26$ \\
\hline $22: 6 n-3^{*}$ & $27.34 \pm 2.10$ & $25.87 \pm 0.99$ & $18.49 \pm 0.65$ & $18.37 \pm 0.68$ & $29.83 \pm 1.30$ & $18.35 \pm 1.11$ \\
\hline $24: 1$ & $1.13 \pm 0.09$ & $1.16 \pm 0.04$ & $1.13 \pm 0.03$ & $0.86 \pm 0.08$ & $0.92 \pm 0.03$ & $0.58 \pm 0.07$ \\
\hline Total SAT & $22.12 \pm 0.41$ & $25.83 \pm 0.39$ & $23.33 \pm 0.31$ & $24.30 \pm 0.30$ & $26.55 \pm 0.39$ & $23.81 \pm 0.21$ \\
\hline Total MUFA & $28.31 \pm 2.03$ & $24.26 \pm 1.03$ & $36.35 \pm 0.73$ & $35.67 \pm 0.85$ & $19.95 \pm 1.06$ & $25.57 \pm 0.55$ \\
\hline \multirow[t]{3}{*}{ Total PUFA } & $49.57 \pm 1.87$ & $49.91 \pm 0.82$ & $40.32 \pm 0.61$ & $40.03 \pm 0.70$ & $53.50 \pm 1.42$ & $50.62 \pm 0.61$ \\
\hline & \multirow{2}{*}{$\begin{array}{c}\text { Greenlings } \\
\text { Hexagrammos sp. }\end{array}$} & \multirow{2}{*}{\multicolumn{3}{|c|}{$\begin{array}{c}\text { Herrings } \\
\text { Pacific herring }\end{array}$}} & Rockfishes & Sablefishes \\
\hline & & & & & Sebastes sp. & Blackcod \\
\hline Age/size clas & & $0+$ & $1+$ & $2+$ & & \\
\hline n: & 8 & 220 & 43 & 97 & 2 & 11 \\
\hline \multicolumn{7}{|l|}{ SAT } \\
\hline $14: 0^{*}$ & $2.51 \pm 0.17$ & $6.04 \pm 0.19$ & $6.91 \pm 0.15$ & $7.52 \pm 0.13$ & $3.24 \pm 0.22$ & $4.02 \pm 0.44$ \\
\hline $15: 0$ & $0.37 \pm 0.03$ & $0.49 \pm 0.01$ & $0.41 \pm 0.01$ & $0.30 \pm 0.01$ & $0.34 \pm 0.04$ & $0.49 \pm 0.06$ \\
\hline iso $16: 0$ & $0.19 \pm 0.02$ & $0.15 \pm 0.01$ & $0.07 \pm 0.01$ & $0.06 \pm 0.00$ & $0.17 \pm 0.02$ & $0.14 \pm 0.01$ \\
\hline $16: 0^{*}$ & $16.28 \pm 0.48$ & $20.72 \pm 0.15$ & $19.17 \pm 0.42$ & $14.75 \pm 0.21$ & $17.22 \pm 0.10$ & $16.45 \pm 0.36$ \\
\hline $17: 0$ & $0.42 \pm 0.11$ & $0.28 \pm 0.01$ & $0.18 \pm 0.01$ & $0.14 \pm 0.01$ & $0.32 \pm 0.00$ & $0.31 \pm 0.04$ \\
\hline $18: 0^{*}$ & $3.82 \pm 0.38$ & $2.60 \pm 0.06$ & $1.81 \pm 0.05$ & $1.60 \pm 0.04$ & $4.44 \pm 0.27$ & $4.61 \pm 0.44$ \\
\hline
\end{tabular}


Table 2 (continued)

\begin{tabular}{|c|c|c|c|c|c|c|}
\hline \multirow[b]{2}{*}{ Age/size class: } & \multirow{2}{*}{$\begin{array}{l}\text { Greenlings } \\
\text { Hexagrammos }\end{array}$} & \multicolumn{3}{|c|}{$\begin{array}{c}\text { Herrings } \\
\text { Pacific herring }\end{array}$} & \multirow[t]{2}{*}{$\begin{array}{l}\text { Rockfishes } \\
\text { Sebastes sp. }\end{array}$} & \multirow[t]{2}{*}{$\begin{array}{c}\text { Sablefishes } \\
\text { Blackcod }\end{array}$} \\
\hline & & $0+$ & $1+$ & $2+$ & & \\
\hline \multicolumn{7}{|l|}{ MUFA } \\
\hline $14: 1 n-9$ & $0.13 \pm 0.02$ & $0.36 \pm 0.01$ & $0.37 \pm 0.02$ & $0.23 \pm 0.01$ & $0.17 \pm 0.03$ & $0.13 \pm 0.02$ \\
\hline $16: 1 n-11$ & $0.50 \pm 0.07$ & $0.49 \pm 0.01$ & $0.58 \pm 0.03$ & $0.41 \pm 0.01$ & $0.43 \pm 0.03$ & $0.86 \pm 0.13$ \\
\hline $16: 1 n-9$ & $0.40 \pm 0.03$ & $0.25 \pm 0.01$ & $0.20 \pm 0.01$ & $0.15 \pm 0.00$ & $0.34 \pm 0.09$ & $0.21 \pm 0.01$ \\
\hline $16: 1 \mathrm{n}-7^{*}$ & $8.42 \pm 1.06$ & $5.80 \pm 0.13$ & $6.02 \pm 0.17$ & $5.29 \pm 0.15$ & $7.63 \pm 1.32$ & $5.67 \pm 0.54$ \\
\hline $17: 1$ & $0.33 \pm 0.02$ & $0.23 \pm 0.01$ & $0.17 \pm 0.01$ & $0.19 \pm 0.01$ & $0.38 \pm 0.01$ & $0.35 \pm 0.08$ \\
\hline $18: 1 n-11$ & $0.19 \pm 0.04$ & $0.09 \pm 0.01$ & $0.18 \pm 0.02$ & $0.53 \pm 0.02$ & $0.32 \pm 0.11$ & $0.25 \pm 0.05$ \\
\hline $18: 1 n-9^{*}$ & $12.61 \pm 1.21$ & $11.29 \pm 0.21$ & $14.81 \pm 0.60$ & $11.65 \pm 0.40$ & $15.31 \pm 1.02$ & $11.16 \pm 0.81$ \\
\hline $18: 1 n-7^{*}$ & $5.14 \pm 0.22$ & $2.74 \pm 0.04$ & $2.28 \pm 0.05$ & $2.30 \pm 0.07$ & $4.57 \pm 0.71$ & $4.66 \pm 0.22$ \\
\hline $18: 1 n-5$ & $0.36 \pm 0.04$ & $0.59 \pm 0.01$ & $0.75 \pm 0.03$ & $0.57 \pm 0.01$ & $0.50 \pm 0.11$ & $0.40 \pm 0.03$ \\
\hline $20: 1 n-11^{*}$ & $1.06 \pm 0.17$ & $1.61 \pm 0.17$ & $2.73 \pm 0.38$ & $10.03 \pm 0.41$ & $1.56 \pm 0.52$ & $2.23 \pm 0.62$ \\
\hline $20: 1 n-9^{*}$ & $0.69 \pm 0.02$ & $0.90 \pm 0.04$ & $2.10 \pm 0.18$ & $3.06 \pm 0.11$ & $1.05 \pm 0.06$ & $1.25 \pm 0.08$ \\
\hline $20: 1 n-7$ & $0.86 \pm 0.09$ & $0.19 \pm 0.01$ & $0.22 \pm 0.01$ & $0.24 \pm 0.01$ & $0.54 \pm 0.17$ & $0.84 \pm 0.09$ \\
\hline $22: 1 \mathrm{n}-11^{*}$ & $0.36 \pm 0.10$ & $1.97 \pm 0.18$ & $4.33 \pm 0.53$ & $12.55 \pm 0.43$ & $1.08 \pm 0.42$ & $1.70 \pm 0.49$ \\
\hline $22: 1 \mathrm{n}-9^{*}$ & $0.12 \pm 0.03$ & $0.21 \pm 0.01$ & $0.45 \pm 0.05$ & $0.57 \pm 0.02$ & $0.33 \pm 0.08$ & $0.34 \pm 0.04$ \\
\hline \multicolumn{7}{|l|}{ PUFA } \\
\hline $16: 2 n-4$ & $0.53 \pm 0.05$ & $0.43 \pm 0.01$ & $0.29 \pm 0.04$ & $0.34 \pm 0.02$ & $0.41 \pm 0.01$ & $0.63 \pm 0.09$ \\
\hline $16: 3 n-6$ & $0.30 \pm 0.03$ & $0.33 \pm 0.01$ & $0.19 \pm 0.04$ & $0.37 \pm 0.02$ & $0.28 \pm 0.04$ & $0.39 \pm 0.06$ \\
\hline $16: 3 n-4$ & $0.22 \pm 0.02$ & $0.28 \pm 0.01$ & $0.29 \pm 0.02$ & $0.30 \pm 0.02$ & $0.26 \pm 0.04$ & $0.24 \pm 0.04$ \\
\hline $16: 4 n-1^{*}$ & $0.30 \pm 0.06$ & $0.53 \pm 0.04$ & $0.53 \pm 0.05$ & $0.51 \pm 0.04$ & $0.34 \pm 0.02$ & $0.43 \pm 0.09$ \\
\hline $18: 2 n-6^{*}$ & $1.88 \pm 0.65$ & $1.13 \pm 0.03$ & $1.09 \pm 0.03$ & $0.82 \pm 0.02$ & $0.82 \pm 0.16$ & $0.84 \pm 0.05$ \\
\hline $18: 3 n-3^{*}$ & $1.46 \pm 0.71$ & $0.93 \pm 0.03$ & $1.23 \pm 0.06$ & $0.65 \pm 0.03$ & $0.48 \pm 0.21$ & $0.53 \pm 0.05$ \\
\hline $18: 4 n-3^{*}$ & $1.48 \pm 0.52$ & $1.99 \pm 0.06$ & $2.85 \pm 0.17$ & $1.55 \pm 0.08$ & $1.15 \pm 0.35$ & $1.03 \pm 0.13$ \\
\hline $20: 2 n-6$ & $0.47 \pm 0.07$ & $0.21 \pm 0.01$ & $0.19 \pm 0.01$ & $0.15 \pm 0.00$ & $0.26 \pm 0.01$ & $0.35 \pm 0.03$ \\
\hline $20: 4 n-6^{*}$ & $2.91 \pm 0.37$ & $0.69 \pm 0.02$ & $0.40 \pm 0.02$ & $0.40 \pm 0.02$ & $1.86 \pm 0.05$ & $1.98 \pm 0.29$ \\
\hline $20: 4 n-3$ & $0.62 \pm 0.11$ & $0.55 \pm 0.01$ & $0.79 \pm 0.03$ & $0.45 \pm 0.01$ & $0.44 \pm 0.06$ & $0.56 \pm 0.04$ \\
\hline $20: 5 n-3^{*}$ & $14.04 \pm 0.68$ & $11.82 \pm 0.16$ & $10.03 \pm 0.25$ & $7.35 \pm 0.17$ & $10.49 \pm 1.06$ & $13.29 \pm 0.64$ \\
\hline $21: 5 n-3$ & $0.35 \pm 0.04$ & $0.27 \pm 0.01$ & $0.31 \pm 0.01$ & $0.23 \pm 0.01$ & $0.26 \pm 0.04$ & $0.35 \pm 0.04$ \\
\hline $22: 5 n-6$ & $0.26 \pm 0.05$ & $0.19 \pm 0.00$ & $0.14 \pm 0.01$ & $0.11 \pm 0.00$ & $0.29 \pm 0.04$ & $0.28 \pm 0.03$ \\
\hline $22: 5 n-3$ & $2.33 \pm 0.27$ & $0.81 \pm 0.01$ & $0.67 \pm 0.02$ & $0.81 \pm 0.02$ & $1.70 \pm 0.36$ & $2.36 \pm 0.23$ \\
\hline $22: 6 n-3^{*}$ & $14.04 \pm 1.35$ & $19.28 \pm 0.52$ & $13.50 \pm 0.43$ & $10.69 \pm 0.32$ & $17.08 \pm 3.03$ & $17.08 \pm 1.38$ \\
\hline $24: 1$ & $0.48 \pm 0.08$ & $0.97 \pm 0.02$ & $0.98 \pm 0.03$ & $0.85 \pm 0.03$ & $0.76 \pm 0.12$ & $0.62 \pm 0.05$ \\
\hline Total SAT & $24.53 \pm 0.36$ & $31.21 \pm 0.19$ & $29.72 \pm 0.44$ & $25.22 \pm 0.17$ & $26.55 \pm 0.04$ & $26.93 \pm 0.45$ \\
\hline Total MUFA & $31.90 \pm 2.07$ & $27.19 \pm 0.51$ & $35.62 \pm 0.89$ & $48.20 \pm 0.45$ & $35.01 \pm 2.31$ & $30.47 \pm 1.84$ \\
\hline \multirow[t]{3}{*}{ Total PUFA } & $43.57 \pm 1.92$ & $41.60 \pm 0.59$ & $34.66 \pm 0.68$ & $26.57 \pm 0.37$ & $38.44 \pm 2.35$ & $42.60 \pm 1.89$ \\
\hline & \multicolumn{6}{|c|}{ Righteye flounders } \\
\hline & Flathead sole & Halibut & Rex sole & Rock sole & Yellowfin sole & Pleuronectidae \\
\hline $\begin{array}{l}\text { Age/size class: } \\
\text { n: }\end{array}$ & 33 & 11 & 23 & 1 & 17 & 24 \\
\hline \multicolumn{7}{|l|}{ SAT } \\
\hline $14: 0^{*}$ & $2.45 \pm 0.13$ & 0.79 & $2.18 \pm 0.19$ & 3.21 & $3.91 \pm 0.22$ & $2.19 \pm 0.12$ \\
\hline $15: 0$ & $0.41 \pm 0.01$ & 0.19 & $0.78 \pm 0.03$ & 0.59 & $0.56 \pm 0.02$ & $0.40 \pm 0.02$ \\
\hline iso $16: 0$ & $0.60 \pm 0.02$ & 0.40 & $0.68 \pm 0.03$ & 0.80 & $0.37 \pm 0.05$ & $0.79 \pm 0.04$ \\
\hline $16: 0^{*}$ & $15.46 \pm 0.11$ & 16.54 & $14.73 \pm 0.17$ & 13.24 & $12.94 \pm 0.20$ & $15.10 \pm 0.18$ \\
\hline $17: 0$ & $0.56 \pm 0.07$ & 0.25 & $0.92 \pm 0.09$ & 0.52 & $0.48 \pm 0.04$ & $0.28 \pm 0.02$ \\
\hline $18: 0^{*}$ & $4.09 \pm 0.10$ & 6.62 & $4.90 \pm 0.16$ & 4.26 & $3.25 \pm 0.16$ & $4.98 \pm 0.16$ \\
\hline \multicolumn{7}{|l|}{ MUFA } \\
\hline $14: 1 n-9$ & $0.22 \pm 0.02$ & 0.10 & $0.10 \pm 0.01$ & 0.12 & $0.35 \pm 0.04$ & $0.24 \pm 0.03$ \\
\hline $16: 1 n-11$ & $0.54 \pm 0.03$ & 0.36 & $0.67 \pm 0.03$ & 0.78 & $0.88 \pm 0.04$ & $0.44 \pm 0.01$ \\
\hline $16: 1 n-9$ & $0.35 \pm 0.01$ & 0.20 & $0.38 \pm 0.01$ & 0.53 & $0.38 \pm 0.01$ & $0.37 \pm 0.02$ \\
\hline $16: 1 \mathrm{n}-7^{*}$ & $7.85 \pm 0.56$ & 1.55 & $7.21 \pm 0.66$ & 6.45 & $10.37 \pm 0.98$ & $5.15 \pm 0.46$ \\
\hline $17: 1$ & $0.26 \pm 0.03$ & 0.20 & $0.48 \pm 0.02$ & 0.40 & $0.38 \pm 0.02$ & $0.31 \pm 0.04$ \\
\hline $18: 1 n-11$ & $0.16 \pm 0.02$ & 0.31 & $0.14 \pm 0.01$ & 0.10 & $0.38 \pm 0.06$ & $0.16 \pm 0.01$ \\
\hline $18: 1 \mathrm{n}-9^{*}$ & $8.85 \pm 0.35$ & 9.80 & $5.07 \pm 0.25$ & 6.56 & $7.46 \pm 0.95$ & $8.75 \pm 0.29$ \\
\hline $18: 1 \mathrm{n}-7^{*}$ & $5.48 \pm 0.16$ & 4.59 & $5.37 \pm 0.26$ & 4.98 & $4.34 \pm 0.20$ & $4.61 \pm 0.17$ \\
\hline $18: 1 n-5$ & $0.64 \pm 0.03$ & 0.25 & $0.36 \pm 0.04$ & 0.46 & $0.41 \pm 0.04$ & $0.34 \pm 0.02$ \\
\hline $20: 1 \mathrm{n}-11^{*}$ & $1.08 \pm 0.08$ & 0.54 & $1.42 \pm 0.11$ & 1.65 & $4.09 \pm 0.81$ & $1.31 \pm 0.11$ \\
\hline $20: 1 n-9^{*}$ & $1.02 \pm 0.04$ & 0.91 & $0.81 \pm 0.06$ & 1.41 & $1.40 \pm 0.16$ & $1.09 \pm 0.05$ \\
\hline
\end{tabular}


Table 2 (continued)

\begin{tabular}{|c|c|c|c|c|c|c|}
\hline & & & Righte & ounders & & \\
\hline & Flathead sole & Halibut & Rex sole & Rock sole & Yellowfin sole & Pleuronectidae \\
\hline $20: 1 n-7$ & $1.14 \pm 0.09$ & 0.34 & $2.57 \pm 0.17$ & 1.79 & $2.05 \pm 0.16$ & $1.08 \pm 0.13$ \\
\hline $22: 1 \mathrm{n}-11^{*}$ & $0.49 \pm 0.08$ & 0.27 & $0.42 \pm 0.03$ & 0.45 & $2.13 \pm 0.52$ & $0.63 \pm 0.07$ \\
\hline $22: 1 n-9^{*}$ & $0.17 \pm 0.01$ & 0.15 & $0.15 \pm 0.02$ & 0.40 & $0.26 \pm 0.04$ & $0.15 \pm 0.01$ \\
\hline PUFA & & & & & & \\
\hline $16: 2 n-4$ & $0.35 \pm 0.03$ & 0.57 & $0.62 \pm 0.06$ & 0.61 & $0.64 \pm 0.04$ & $0.23 \pm 0.03$ \\
\hline $16: 3 n-6$ & $0.44 \pm 0.02$ & 0.05 & $0.34 \pm 0.05$ & 0.37 & $0.42 \pm 0.03$ & $0.40 \pm 0.03$ \\
\hline $16: 3 n-4$ & $0.34 \pm 0.02$ & 0.04 & $0.19 \pm 0.02$ & 0.35 & $0.31 \pm 0.03$ & $0.22 \pm 0.04$ \\
\hline $16: 4 n-1^{*}$ & $0.73 \pm 0.04$ & 0.38 & $0.45 \pm 0.02$ & 0.48 & $0.50 \pm 0.07$ & $0.72 \pm 0.04$ \\
\hline $18: 2 n-6^{*}$ & $0.75 \pm 0.02$ & 0.50 & $0.49 \pm 0.06$ & 0.65 & $0.78 \pm 0.04$ & $0.77 \pm 0.03$ \\
\hline $18: 3 n-3^{*}$ & $0.32 \pm 0.03$ & 0.07 & $0.15 \pm 0.02$ & 0.16 & $0.38 \pm 0.05$ & $0.26 \pm 0.03$ \\
\hline $18: 4 n-3^{*}$ & $0.90 \pm 0.07$ & 0.07 & $0.54 \pm 0.07$ & 0.90 & $1.09 \pm 0.07$ & $0.79 \pm 0.11$ \\
\hline $20: 2 n-6$ & $0.26 \pm 0.02$ & 0.27 & $0.43 \pm 0.03$ & 0.55 & $0.39 \pm 0.02$ & $0.20 \pm 0.01$ \\
\hline $20: 4 n-6^{*}$ & $3.11 \pm 0.19$ & 3.44 & $5.02 \pm 0.23$ & 4.10 & $2.58 \pm 0.19$ & $5.09 \pm 0.32$ \\
\hline $20: 4 n-3$ & $0.39 \pm 0.02$ & 0.26 & $0.34 \pm 0.02$ & 0.52 & $0.47 \pm 0.02$ & $0.32 \pm 0.03$ \\
\hline $20: 5 n-3^{*}$ & $15.02 \pm 0.41$ & 9.92 & $15.10 \pm 0.36$ & 17.80 & $14.60 \pm 1.30$ & $12.23 \pm 0.33$ \\
\hline $21: 5 n-3$ & $0.34 \pm 0.02$ & 0.16 & $0.33 \pm 0.03$ & 0.47 & $0.37 \pm 0.04$ & $0.22 \pm 0.02$ \\
\hline $22: 5 n-6$ & $0.47 \pm 0.03$ & 0.50 & $0.81 \pm 0.05$ & 0.47 & $0.41 \pm 0.03$ & $0.58 \pm 0.02$ \\
\hline $22: 5 n-3$ & $2.70 \pm 0.07$ & 4.84 & $3.79 \pm 0.09$ & 3.30 & $3.18 \pm 0.11$ & $2.50 \pm 0.06$ \\
\hline $22: 6 n-3^{*}$ & $17.90 \pm 0.85$ & 31.24 & $16.73 \pm 0.71$ & 13.67 & $12.60 \pm 0.69$ & $22.86 \pm 0.90$ \\
\hline $24: 1$ & $0.72 \pm 0.04$ & 1.20 & $0.50 \pm 0.04$ & 0.62 & $0.50 \pm 0.06$ & $1.20 \pm 0.07$ \\
\hline Total SAT & $24.61 \pm 0.11$ & 25.28 & $25.24 \pm 0.18$ & 24.32 & $22.51 \pm 0.25$ & $24.47 \pm 0.20$ \\
\hline Total MUFA & $29.13 \pm 0.53$ & 19.99 & $26.69 \pm 0.72$ & 27.83 & $36.03 \pm 1.26$ & $25.25 \pm 0.77$ \\
\hline Total PUFA & $46.26 \pm 0.56$ & 54.73 & $48.07 \pm 0.63$ & 47.85 & $41.46 \pm 1.11$ & $50.28 \pm 0.71$ \\
\hline & & Salmonids & & - Sa & lances & Sculpins \\
\hline & Chum salmon & Pink & lmon & Pacifi & sandlance & Cottidae \\
\hline Age/size class: & & Smolts & Adults & $0+$ & $1+$ & \\
\hline $\mathrm{n}$ & 7 & 40 & 10 & 66 & 41 & 7 \\
\hline SAT & & & & & & \\
\hline $14: 0^{*}$ & $1.35 \pm 0.21$ & $2.00 \pm 0.10$ & $3.39 \pm 0.17$ & $4.85 \pm 0.15$ & $6.06 \pm 0.27$ & $1.59 \pm 0.24$ \\
\hline $15: 0$ & $0.23 \pm 0.01$ & $0.58 \pm 0.02$ & $0.43 \pm 0.02$ & $0.57 \pm 0.02$ & $0.40 \pm 0.01$ & $0.39 \pm 0.05$ \\
\hline iso $16: 0$ & $0.38 \pm 0.02$ & $0.54 \pm 0.02$ & $0.14 \pm 0.01$ & $0.21 \pm 0.01$ & $0.17 \pm 0.01$ & $0.24 \pm 0.03$ \\
\hline $16: 0^{*}$ & $21.37 \pm 0.32$ & $18.47 \pm 0.13$ & $12.67 \pm 0.50$ & $18.68 \pm 0.22$ & $19.07 \pm 0.36$ & $15.17 \pm 0.38$ \\
\hline $17: 0$ & $0.33 \pm 0.01$ & $0.47 \pm 0.01$ & $0.29 \pm 0.04$ & $0.43 \pm 0.02$ & $0.28 \pm 0.02$ & $0.42 \pm 0.05$ \\
\hline $18: 0^{*}$ & $5.23 \pm 0.16$ & $4.81 \pm 0.11$ & $3.75 \pm 0.15$ & $3.50 \pm 0.08$ & $2.54 \pm 0.09$ & $5.45 \pm 0.31$ \\
\hline MUFA & & & & & & \\
\hline $14: 1 n-9$ & $0.05 \pm 0.01$ & $0.05 \pm 0.00$ & $0.05 \pm 0.00$ & $0.31 \pm 0.01$ & $0.32 \pm 0.02$ & $0.11 \pm 0.03$ \\
\hline $16: 1 n-11$ & $0.29 \pm 0.01$ & $0.57 \pm 0.03$ & $0.40 \pm 0.02$ & $0.66 \pm 0.01$ & $0.51 \pm 0.01$ & $0.38 \pm 0.03$ \\
\hline $16: 1 n-9$ & $0.65 \pm 0.05$ & $0.40 \pm 0.02$ & $0.26 \pm 0.01$ & $0.27 \pm 0.01$ & $0.18 \pm 0.01$ & $0.43 \pm 0.03$ \\
\hline $16: 1 \mathrm{n}-7^{*}$ & $2.09 \pm 0.13$ & $2.33 \pm 0.17$ & $4.22 \pm 0.21$ & $3.96 \pm 0.15$ & $6.22 \pm 0.29$ & $5.14 \pm 0.79$ \\
\hline $17: 1$ & $0.16 \pm 0.00$ & $0.09 \pm 0.01$ & $0.15 \pm 0.05$ & $0.18 \pm 0.00$ & $0.19 \pm 0.01$ & $0.40 \pm 0.06$ \\
\hline 18:1n-11 & $0.07 \pm 0.01$ & $0.13 \pm 0.02$ & $0.87 \pm 0.11$ & $0.09 \pm 0.01$ & $0.17 \pm 0.03$ & $0.29 \pm 0.08$ \\
\hline $18: 1 n-9^{*}$ & $9.16 \pm 0.23$ & $6.63 \pm 0.18$ & $11.98 \pm 0.47$ & $7.96 \pm 0.14$ & $11.59 \pm 0.60$ & $12.95 \pm 1.40$ \\
\hline $18: 1 \mathrm{n}-7^{*}$ & $2.79 \pm 0.10$ & $2.30 \pm 0.07$ & $2.47 \pm 0.17$ & $1.90 \pm 0.04$ & $2.25 \pm 0.08$ & $5.43 \pm 0.73$ \\
\hline $18: 1 n-5$ & $0.42 \pm 0.03$ & $0.40 \pm 0.02$ & $0.61 \pm 0.04$ & $0.66 \pm 0.02$ & $0.58 \pm 0.03$ & $0.39 \pm 0.03$ \\
\hline $20: 1 \mathrm{n}-11^{*}$ & $0.21 \pm 0.06$ & $0.35 \pm 0.08$ & $2.91 \pm 0.25$ & $0.54 \pm 0.18$ & $2.02 \pm 0.44$ & $0.73 \pm 0.14$ \\
\hline $20: 1 n-9^{*}$ & $0.46 \pm 0.04$ & $0.52 \pm 0.06$ & $1.79 \pm 0.09$ & $0.91 \pm 0.07$ & $1.38 \pm 0.17$ & $0.79 \pm 0.04$ \\
\hline $20: 1 n-7$ & $0.15 \pm 0.02$ & $0.22 \pm 0.01$ & $0.42 \pm 0.07$ & $0.20 \pm 0.02$ & $0.34 \pm 0.05$ & $0.61 \pm 0.17$ \\
\hline $22: 1 n-11^{*}$ & $0.12 \pm 0.02$ & $0.27 \pm 0.06$ & $4.30 \pm 0.48$ & $0.83 \pm 0.22$ & $2.57 \pm 0.50$ & $0.26 \pm 0.06$ \\
\hline $22: 1 n-9^{*}$ & $0.08 \pm 0.02$ & $0.12 \pm 0.02$ & $0.48 \pm 0.05$ & $0.29 \pm 0.02$ & $0.27 \pm 0.02$ & $0.13 \pm 0.03$ \\
\hline PUFA & & & & & & \\
\hline $16: 2 n-4$ & $0.40 \pm 0.04$ & $0.63 \pm 0.04$ & $0.34 \pm 0.02$ & $0.85 \pm 0.04$ & $0.51 \pm 0.01$ & $0.47 \pm 0.09$ \\
\hline $16: 3 n-6$ & $0.06 \pm 0.01$ & $0.06 \pm 0.01$ & $0.18 \pm 0.01$ & $0.26 \pm 0.01$ & $0.37 \pm 0.03$ & $0.19 \pm 0.05$ \\
\hline $16: 3 n-4$ & $0.07 \pm 0.01$ & $0.13 \pm 0.01$ & $0.24 \pm 0.06$ & $0.18 \pm 0.01$ & $0.38 \pm 0.03$ & $0.15 \pm 0.03$ \\
\hline $16: 4 n-1^{*}$ & $0.29 \pm 0.01$ & $0.24 \pm 0.01$ & $0.10 \pm 0.01$ & $0.28 \pm 0.02$ & $0.78 \pm 0.06$ & $0.10 \pm 0.04$ \\
\hline $18: 2 n-6^{*}$ & $1.35 \pm 0.17$ & $1.32 \pm 0.09$ & $1.34 \pm 0.04$ & $1.65 \pm 0.03$ & $1.35 \pm 0.08$ & $1.64 \pm 0.29$ \\
\hline $18: 3 n-3^{*}$ & $0.68 \pm 0.07$ & $1.13 \pm 0.07$ & $1.05 \pm 0.06$ & $1.56 \pm 0.04$ & $1.08 \pm 0.06$ & $0.75 \pm 0.15$ \\
\hline $18: 4 n-3^{*}$ & $0.61 \pm 0.07$ & $0.89 \pm 0.06$ & $1.86 \pm 0.13$ & $3.23 \pm 0.07$ & $2.72 \pm 0.17$ & $0.55 \pm 0.09$ \\
\hline
\end{tabular}


Table 2 (continued)

\begin{tabular}{|c|c|c|c|c|c|c|}
\hline \multirow[b]{2}{*}{ Age/size class: } & \multirow{2}{*}{ Chum salmon } & \multicolumn{2}{|c|}{$\begin{array}{l}\text { Salmonids } \\
\text { Pink salmon }\end{array}$} & \multicolumn{2}{|c|}{$\begin{array}{l}\text { Sandlances } \\
\text { Pacific sandlance }\end{array}$} & \multirow[t]{2}{*}{$\begin{array}{l}\text { Sculpins } \\
\text { Cottidae }\end{array}$} \\
\hline & & Smolts & Adults & $0+$ & $1+$ & \\
\hline $20: 2 n-6$ & $0.24 \pm 0.02$ & $0.29 \pm 0.01$ & $0.38 \pm 0.03$ & $0.34 \pm 0.01$ & $0.27 \pm 0.02$ & $0.43 \pm 0.04$ \\
\hline $14: 0^{*}$ & $1.35 \pm 0.21$ & $2.00 \pm 0.10$ & $3.39 \pm 0.17$ & $4.85 \pm 0.15$ & $6.06 \pm 0.27$ & $1.59 \pm 0.24$ \\
\hline $20: 4 n-6^{*}$ & $2.04 \pm 0.25$ & $1.49 \pm 0.04$ & $1.02 \pm 0.03$ & $0.93 \pm 0.03$ & $0.55 \pm 0.05$ & $4.04 \pm 0.37$ \\
\hline $20: 4 n-3$ & $0.63 \pm 0.04$ & $1.10 \pm 0.04$ & $1.91 \pm 0.14$ & $0.80 \pm 0.01$ & $0.63 \pm 0.02$ & $0.72 \pm 0.11$ \\
\hline $20: 5 n-3^{*}$ & $10.19 \pm 0.50$ & $8.78 \pm 0.26$ & $12.41 \pm 0.45$ & $13.83 \pm 0.17$ & $13.07 \pm 0.36$ & $13.58 \pm 1.09$ \\
\hline $21: 5 n-3$ & $0.19 \pm 0.01$ & $0.15 \pm 0.01$ & $0.38 \pm 0.02$ & $0.34 \pm 0.01$ & $0.35 \pm 0.02$ & $0.27 \pm 0.07$ \\
\hline $22: 5 n-6$ & $0.45 \pm 0.03$ & $0.43 \pm 0.04$ & $0.15 \pm 0.01$ & $0.29 \pm 0.01$ & $0.19 \pm 0.01$ & $0.27 \pm 0.02$ \\
\hline $22: 5 n-3$ & $2.53 \pm 0.05$ & $2.54 \pm 0.03$ & $4.19 \pm 0.25$ & $0.78 \pm 0.02$ & $0.81 \pm 0.03$ & $3.99 \pm 0.19$ \\
\hline $22: 6 n-3^{*}$ & $31.82 \pm 1.17$ & $36.14 \pm 0.60$ & $19.69 \pm 0.52$ & $23.33 \pm 0.55$ & $15.95 \pm 0.71$ & $17.68 \pm 2.10$ \\
\hline $24: 1$ & $0.86 \pm 0.03$ & $0.72 \pm 0.03$ & $0.61 \pm 0.04$ & $1.14 \pm 0.02$ & $1.06 \pm 0.05$ & $0.55 \pm 0.05$ \\
\hline Total SAT & $29.32 \pm 0.34$ & $27.56 \pm 0.11$ & $21.54 \pm 0.50$ & $29.39 \pm 0.31$ & $29.42 \pm 0.45$ & $24.20 \pm 0.41$ \\
\hline Total MUFA & $16.90 \pm 0.57$ & $14.76 \pm 0.44$ & $31.35 \pm 0.72$ & $19.35 \pm 0.50$ & $29.16 \pm 0.76$ & $28.61 \pm 1.60$ \\
\hline \multirow[t]{3}{*}{ Total PUFA } & $53.78 \pm 0.81$ & $57.67 \pm 0.43$ & $47.10 \pm 0.69$ & $51.26 \pm 0.60$ & $41.42 \pm 0.60$ & $47.19 \pm 1.44$ \\
\hline & \multicolumn{3}{|r|}{ Squid } & & & Octopus \\
\hline & & agister armnoc & & North Paciflc bobtall & Gonatidae & Uctopus sp. \\
\hline Age/size class: & Small & Medium & Large & & & \\
\hline $\mathrm{n}:$ & 8 & 2 & 2 & 22 & 83 & 7 \\
\hline \multicolumn{7}{|l|}{ SAT } \\
\hline $14: 0^{*}$ & $5.60 \pm 0.61$ & $2.52 \pm 0.89$ & $4.71 \pm 0.38$ & $2.55 \pm 0.07$ & $2.41 \pm 0.10$ & $1.49 \pm 0.14$ \\
\hline 15:0 & $0.42 \pm 0.03$ & $0.24 \pm 0.07$ & $0.21 \pm 0.02$ & $0.44 \pm 0.01$ & $0.37 \pm 0.03$ & $0.32 \pm 0.04$ \\
\hline iso $16: 0$ & $0.36 \pm 0.03$ & $0.27 \pm 0.07$ & $0.10 \pm 0.02$ & $0.67 \pm 0.02$ & $0.62 \pm 0.02$ & $0.83 \pm 0.13$ \\
\hline $16: 0^{*}$ & $18.05 \pm 1.35$ & $15.84 \pm 1.05$ & $10.24 \pm 0.29$ & $14.14 \pm 0.25$ & $19.11 \pm 0.27$ & $15.43 \pm 0.68$ \\
\hline $17: 0$ & $0.17 \pm 0.02$ & $0.17 \pm 0.01$ & $0.13 \pm 0.01$ & $0.55 \pm 0.02$ & $0.50 \pm 0.07$ & $0.85 \pm 0.10$ \\
\hline $18: 0^{*}$ & $1.71 \pm 0.05$ & $2.05 \pm 0.09$ & $1.84 \pm 0.08$ & $3.62 \pm 0.08$ & $2.18 \pm 0.03$ & $4.53 \pm 0.45$ \\
\hline \multicolumn{7}{|l|}{ MUFA } \\
\hline $14: 1 n-9$ & $0.13 \pm 0.01$ & $0.14 \pm 0.03$ & $0.20 \pm 0.01$ & $0.15 \pm 0.01$ & $0.11 \pm 0.00$ & $0.11 \pm 0.04$ \\
\hline $16: 1 n-11$ & $0.53 \pm 0.03$ & $0.30 \pm 0.11$ & $0.30 \pm 0.04$ & $0.59 \pm 0.02$ & $0.28 \pm 0.02$ & $0.40 \pm 0.04$ \\
\hline $16: 1 n-9$ & $0.20 \pm 0.01$ & $0.17 \pm 0.01$ & $0.27 \pm 0.03$ & $1.06 \pm 0.07$ & $0.11 \pm 0.00$ & $0.17 \pm 0.03$ \\
\hline $16: 1 \mathrm{n}-7^{*}$ & $5.34 \pm 0.50$ & $2.79 \pm 0.86$ & $5.43 \pm 0.76$ & $3.64 \pm 0.25$ & $3.18 \pm 0.26$ & $2.33 \pm 0.59$ \\
\hline $17: 1$ & $0.25 \pm 0.03$ & $0.17 \pm 0.03$ & $0.17 \pm 0.00$ & $0.29 \pm 0.02$ & $0.08 \pm 0.01$ & $0.09 \pm 0.02$ \\
\hline $18: 1 n-11$ & $0.15 \pm 0.03$ & $0.63 \pm 0.35$ & $0.86 \pm 0.04$ & $0.52 \pm 0.05$ & $0.31 \pm 0.03$ & $0.17 \pm 0.03$ \\
\hline $18: 1 n-9^{*}$ & $13.17 \pm 1.83$ & $13.85 \pm 0.43$ & $13.96 \pm 3.54$ & $4.06 \pm 0.22$ & $9.37 \pm 0.43$ & $3.32 \pm 0.80$ \\
\hline $18: 1 \mathrm{n}-7^{*}$ & $5.41 \pm 0.26$ & $4.66 \pm 0.17$ & $3.14 \pm 0.07$ & $4.47 \pm 0.23$ & $3.91 \pm 0.18$ & $4.17 \pm 0.37$ \\
\hline $18: 1 n-5$ & $0.37 \pm 0.04$ & $0.53 \pm 0.03$ & $0.53 \pm 0.05$ & $0.53 \pm 0.01$ & $0.55 \pm 0.02$ & $0.47 \pm 0.02$ \\
\hline $20: 1 n-11^{*}$ & $1.89 \pm 0.29$ & $4.10 \pm 0.81$ & $7.20 \pm 1.10$ & $3.46 \pm 0.46$ & $2.31 \pm 0.21$ & $1.52 \pm 0.55$ \\
\hline $20: 1 \mathrm{n}-9^{*}$ & $1.78 \pm 0.13$ & $3.77 \pm 0.47$ & $4.58 \pm 0.18$ & $4.56 \pm 0.18$ & $2.83 \pm 0.09$ & $3.88 \pm 0.31$ \\
\hline $20: 1 n-7$ & $0.34 \pm 0.02$ & $0.32 \pm 0.00$ & $0.29 \pm 0.01$ & $0.69 \pm 0.05$ & $0.18 \pm 0.01$ & $1.03 \pm 0.25$ \\
\hline $22: 1 \mathrm{n}-11^{*}$ & $1.99 \pm 0.41$ & $2.98 \pm 0.06$ & $8.98 \pm 0.09$ & $3.20 \pm 0.59$ & $1.52 \pm 0.15$ & $1.16 \pm 0.51$ \\
\hline $22: 1 n-9^{*}$ & $0.52 \pm 0.05$ & $0.56 \pm 0.00$ & $0.89 \pm 0.03$ & $0.54 \pm 0.02$ & $0.39 \pm 0.02$ & $0.77 \pm 0.10$ \\
\hline \multicolumn{7}{|l|}{ PUFA } \\
\hline $16: 2 n-4$ & $0.13 \pm 0.02$ & $0.33 \pm 0.03$ & $0.41 \pm 0.12$ & $0.20 \pm 0.02$ & $0.20 \pm 0.01$ & $0.09 \pm 0.051$ \\
\hline $16: 3 n-6$ & $0.28 \pm 0.09$ & $0.36 \pm 0.06$ & $0.10 \pm 0.01$ & $0.22 \pm 0.02$ & $0.06 \pm 0.02$ & $0.53 \pm 0.04$ \\
\hline $16: 3 n-4$ & $0.31 \pm 0.05$ & $0.11 \pm 0.07$ & $0.24 \pm 0.03$ & $0.04 \pm 0.00$ & $0.08 \pm 0.01$ & $0.10 \pm 0.04$ \\
\hline $16: 4 n-1^{*}$ & $0.40 \pm 0.09$ & $0.11 \pm 0.06$ & $0.31 \pm 0.04$ & $0.07 \pm 0.00$ & $0.14 \pm 0.01$ & $0.39 \pm 0.15$ \\
\hline $18: 2 n-6^{*}$ & $1.25 \pm 0.08$ & $0.98 \pm 0.15$ & $0.96 \pm 0.07$ & $0.52 \pm 0.02$ & $0.74 \pm 0.02$ & $0.59 \pm 0.07$ \\
\hline $18: 3 n-3^{*}$ & $1.10 \pm 0.09$ & $0.90 \pm 0.18$ & $0.61 \pm 0.05$ & $0.36 \pm 0.02$ & $0.32 \pm 0.02$ & $0.21 \pm 0.05$ \\
\hline $18: 4 n-3^{*}$ & $1.78 \pm 0.20$ & $1.15 \pm 0.58$ & $1.31 \pm 0.24$ & $0.45 \pm 0.06$ & $0.58 \pm 0.06$ & $0.29 \pm 0.09$ \\
\hline $20: 2 n-6$ & $0.50 \pm 0.03$ & $0.66 \pm 0.12$ & $0.47 \pm 0.03$ & $0.65 \pm 0.02$ & $0.64 \pm 0.02$ & $0.58 \pm 0.05$ \\
\hline $20: 4 n-6^{*}$ & $0.87 \pm 0.08$ & $0.77 \pm 0.12$ & $0.61 \pm 0.06$ & $2.19 \pm 0.18$ & $0.85 \pm 0.03$ & $5.72 \pm 0.55$ \\
\hline $20: 4 n-3$ & $0.46 \pm 0.03$ & $0.75 \pm 0.02$ & $0.63 \pm 0.00$ & $0.35 \pm 0.02$ & $0.39 \pm 0.02$ & $0.27 \pm 0.04$ \\
\hline $20: 5 n-3^{*}$ & $13.72 \pm 1.22$ & $12.89 \pm 0.51$ & $10.18 \pm 1.23$ & $19.07 \pm 0.41$ & $15.80 \pm 0.19$ & $18.60 \pm 0.66$ \\
\hline $21: 5 n-3$ & $0.37 \pm 0.04$ & $0.41 \pm 0.01$ & $0.32 \pm 0.04$ & $0.27 \pm 0.01$ & $0.39 \pm 0.01$ & $0.48 \pm 0.08$ \\
\hline $22: 5 n-6$ & $0.17 \pm 0.02$ & $0.16 \pm 0.01$ & $0.15 \pm 0.02$ & $0.29 \pm 0.01$ & $0.15 \pm 0.00$ & $0.47 \pm 0.06$ \\
\hline $22: 5 n-3$ & $0.54 \pm 0.04$ & $0.66 \pm 0.05$ & $0.95 \pm 0.07$ & $1.48 \pm 0.05$ & $0.54 \pm 0.01$ & $2.50 \pm 0.33$ \\
\hline $22: 6 n-3^{*}$ & $15.49 \pm 1.16$ & $20.44 \pm 2.52$ & $14.62 \pm 1.73$ & $19.94 \pm 0.49$ & $25.38 \pm 0.92$ & $21.97 \pm 1.00$ \\
\hline $24: 1$ & $0.51 \pm 0.08$ & $0.67 \pm 0.08$ & $0.90 \pm 0.02$ & $0.35 \pm 0.02$ & $0.45 \pm 0.02$ & $0.23 \pm 0.07$ \\
\hline Total SAT & $27.44 \pm 0.97$ & $21.75 \pm 2.05$ & $18.00 \pm 0.87$ & $22.79 \pm 0.31$ & $25.87 \pm 0.29$ & $24.22 \pm 1.10$ \\
\hline Total MUFA & $32.81 \pm 2.88$ & $35.40 \pm 0.16$ & $47.41 \pm 4.02$ & $28.76 \pm 1.07$ & $25.64 \pm 0.90$ & $20.76 \pm 2.24$ \\
\hline Total PUFA & $39.75 \pm 2.54$ & $42.85 \pm 2.21$ & $34.59 \pm 3.15$ & $48.44 \pm 0.79$ & $48.48 \pm 0.97$ & $55.02 \pm 1.47$ \\
\hline
\end{tabular}


Table 2 (continued)

\begin{tabular}{|c|c|c|c|c|}
\hline \multirow{3}{*}{$\begin{array}{l}\text { Age/size class: } \\
\text { n }\end{array}$} & \multicolumn{3}{|c|}{ - Smelts } & \multirow{2}{*}{$\begin{array}{c}\text { Shrimps } \\
\text { Pandalus sp }\end{array}$} \\
\hline & Capelin & Eulachon & Rainbow smelt & \\
\hline & 72 & 20 & 4 & 17 \\
\hline \multicolumn{5}{|l|}{ SAT } \\
\hline $14: 0^{*}$ & $5.12 \pm 0.33$ & $8.62 \pm 0.17$ & $2.61 \pm 0.35$ & $3.02 \pm 0.30$ \\
\hline $15: 0$ & $0.22 \pm 0.01$ & $0.30 \pm 0.01$ & $0.27 \pm 0.03$ & $0.50 \pm 0.02$ \\
\hline iso $16: 0$ & $0.18 \pm 0.01$ & $0.08 \pm 0.00$ & $0.10 \pm 0.03$ & $0.69 \pm 0.04$ \\
\hline $16: 0^{*}$ & $15.43 \pm 0.21$ & $16.68 \pm 0.42$ & $18.23 \pm 0.39$ & $15.86 \pm 0.23$ \\
\hline $17: 0$ & $0.15 \pm 0.01$ & $0.11 \pm 0.00$ & $0.23 \pm 0.04$ & $0.41 \pm 0.03$ \\
\hline $18: 0^{*}$ & $2.45 \pm 0.09$ & $2.43 \pm 0.09$ & $3.82 \pm 0.22$ & $2.29 \pm 0.07$ \\
\hline \multicolumn{5}{|l|}{ MUFA } \\
\hline $14: 1 n-9$ & $0.13 \pm 0.01$ & $0.12 \pm 0.01$ & $0.06 \pm 0.01$ & $0.08 \pm 0.02$ \\
\hline $16: 1 n-11$ & $0.35 \pm 0.01$ & $0.36 \pm 0.01$ & $0.22 \pm 0.02$ & $0.33 \pm 0.01$ \\
\hline $16: 1 n-9$ & $0.12 \pm 0.00$ & $0.27 \pm 0.01$ & $0.34 \pm 0.04$ & $0.20 \pm 0.01$ \\
\hline $16: 1 \mathrm{n}-7^{*}$ & $2.66 \pm 0.16$ & $8.05 \pm 0.37$ & $10.53 \pm 1.65$ & $6.92 \pm 0.38$ \\
\hline $17: 1$ & $0.03 \pm 0.01$ & $0.33 \pm 0.01$ & $0.30 \pm 0.04$ & $0.60 \pm 0.04$ \\
\hline $18: 1 n-11$ & $0.63 \pm 0.02$ & $0.38 \pm 0.05$ & $0.10 \pm 0.02$ & $0.09 \pm 0.01$ \\
\hline $18: 1 n-9^{*}$ & $5.90 \pm 0.17$ & $31.32 \pm 1.01$ & $18.52 \pm 2.62$ & $14.11 \pm 0.28$ \\
\hline $18: 1 \mathrm{n}-7^{*}$ & $2.10 \pm 0.07$ & $5.01 \pm 0.24$ & $4.79 \pm 0.11$ & $6.24 \pm 0.17$ \\
\hline $18: 1 n-5$ & $0.52 \pm 0.01$ & $0.54 \pm 0.02$ & $0.46 \pm 0.04$ & $0.56 \pm 0.02$ \\
\hline $20: 1 n-11^{*}$ & $7.18 \pm 0.46$ & $3.98 \pm 0.43$ & $0.40 \pm 0.08$ & $1.81 \pm 0.22$ \\
\hline $20: 1 n-9^{*}$ & $1.79 \pm 0.10$ & $1.79 \pm 0.09$ & $0.62 \pm 0.14$ & $1.19 \pm 0.13$ \\
\hline $20: 1 n-7$ & $0.19 \pm 0.01$ & $0.20 \pm 0.01$ & $0.25 \pm 0.15$ & $0.91 \pm 0.13$ \\
\hline $22: 1 \mathrm{n}-11^{*}$ & $6.61 \pm 0.53$ & $5.94 \pm 0.67$ & $0.15 \pm 0.01$ & $2.10 \pm 0.35$ \\
\hline $22: 1 \mathrm{n}-9^{*}$ & $0.34 \pm 0.02$ & $0.69 \pm 0.07$ & $0.14 \pm 0.03$ & $0.99 \pm 0.16$ \\
\hline \multicolumn{5}{|l|}{ PUFA } \\
\hline $16: 2 n-4$ & $0.20 \pm 0.01$ & $0.21 \pm 0.01$ & $0.28 \pm 0.11$ & $0.15 \pm 0.01$ \\
\hline $16: 3 n-6$ & $0.26 \pm 0.03$ & $0.49 \pm 0.03$ & $0.23 \pm 0.14$ & $0.21 \pm 0.02$ \\
\hline $16: 3 n-4$ & $0.24 \pm 0.02$ & $0.22 \pm 0.01$ & $0.04 \pm 0.01$ & $0.10 \pm 0.01$ \\
\hline $16: 4 n-1^{*}$ & $0.54 \pm 0.06$ & $0.16 \pm 0.02$ & $0.13 \pm 0.03$ & $0.15 \pm 0.03$ \\
\hline $18: 2 n-6^{*}$ & $0.72 \pm 0.02$ & $0.86 \pm 0.02$ & $0.54 \pm 0.18$ & $0.79 \pm 0.02$ \\
\hline $18: 3 n-3^{*}$ & $0.48 \pm 0.03$ & $0.36 \pm 0.02$ & $0.30 \pm 0.14$ & $0.35 \pm 0.01$ \\
\hline $18: 4 n-3^{*}$ & $1.08 \pm 0.10$ & $0.56 \pm 0.05$ & $0.25 \pm 0.05$ & $0.25 \pm 0.02$ \\
\hline $20: 2 n-6$ & $0.16 \pm 0.01$ & $0.09 \pm 0.00$ & $0.16 \pm 0.02$ & $0.36 \pm 0.03$ \\
\hline $20: 4 n-6^{*}$ & $0.59 \pm 0.03$ & $0.22 \pm 0.01$ & $1.75 \pm 0.47$ & $3.06 \pm 0.20$ \\
\hline $20: 4 n-3$ & $0.42 \pm 0.01$ & $0.13 \pm 0.01$ & $0.20 \pm 0.01$ & $0.38 \pm 0.04$ \\
\hline $20: 5 n-3^{*}$ & $13.18 \pm 0.34$ & $1.52 \pm 0.09$ & $8.85 \pm 0.54$ & $16.25 \pm 1.07$ \\
\hline $21: 5 n-3$ & $0.36 \pm 0.01$ & $0.15 \pm 0.01$ & $0.16 \pm 0.02$ & $0.21 \pm 0.01$ \\
\hline $22: 5 n-6$ & $0.16 \pm 0.01$ & $0.14 \pm 0.02$ & $0.26 \pm 0.06$ & $0.23 \pm 0.01$ \\
\hline $22: 5 n-3$ & $1.54 \pm 0.04$ & $0.21 \pm 0.01$ & $1.48 \pm 0.21$ & $1.19 \pm 0.07$ \\
\hline $22: 6 n-3^{*}$ & $24.66 \pm 1.01$ & $2.47 \pm 0.11$ & $20.35 \pm 2.69$ & $13.19 \pm 0.83$ \\
\hline $24: 1$ & $1.16 \pm 0.04$ & $0.84 \pm 0.05$ & $0.90 \pm 0.11$ & $1.02 \pm 0.06$ \\
\hline Total SAT & $24.56 \pm 0.15$ & $29.12 \pm 0.39$ & $25.54 \pm 0.56$ & $23.78 \pm 0.31$ \\
\hline Total MUFA & $28.81 \pm 1.04$ & $59.59 \pm 0.50$ & $37.36 \pm 3.94$ & $37.00 \pm 1.29$ \\
\hline Total PUFA & $46.63 \pm 1.12$ & $11.29 \pm 0.59$ & $37.10 \pm 4.01$ & $39.22 \pm 1.42$ \\
\hline
\end{tabular}

classified, were most frequently misclassified as one another (Table 3). However, when discriminant analysis was performed on pollock, herring and sandlance alone (Fig. 2), 95.1\% of all individuals were correctly classified.

Classification of species by their fatty acid signatures was also examined using CART analysis, since CART places fewer restrictions on sample size and no restrictions on variable number (i.e. all fatty acids quantified were used). CART analysis of 22 species (with $\mathrm{n} \geq 5$ ) correctly classified $91.8 \%$ of grouped cases (Table 4). In general, classification rates were poorest for those species that had the smallest sample sizes. Thus, when CART analysis was performed using only the same 13 species used in the discriminant analysis (with $\mathrm{n} \geq 17$ ), the percent correctly classified increased to $94.6 \%$ of grouped cases (Table 4). A comparison of classifications obtained using CART and discriminant analysis, showed that CART generally performed better, at 94.6 versus $91.0 \%$ overall. Again, eulachon were $100 \%$ correctly classified, while pollock, herring and capelin were 94 to $96 \%$ correctly classified. CART was significantly better than discriminant analysis in classifying both tomcod (44, compared to 29, of 48 correctly classified) and the 4 groups of flatfish (Table 4), although in the latter case, when flatfish were misclassified they still tended to be misclassified as one another.

Groupings of species in CART were also predictable. For instance, despite some apparent differences among the flatfish species (Table 2), all flatfish were classified together down 1 node of the tree with misclassifications attributed within this node. In contrast , capelin, herring, pollock, sandlance and tomcod generally required more

Pacific tomcod were classified poorly $(60.4 \%$, Table 3$)$ and misclassified most frequently as pollock. Pollock, herring and sandlance were generally well-classified at 91.0, 94.5 and $91.6 \%$ overall. However, these 3 species were also relatively closely associated on the first 2 discriminant functions (Fig. 1) and hence when mis- splits and fatty acids for correct classification and appeared at several points in the tree, suggesting both within-species variability and some between-species similarity (e.g. Figs. 1 \& 2) in signatures among these species. This was also apparent from the results of discriminant analysis (Table 3 ). 

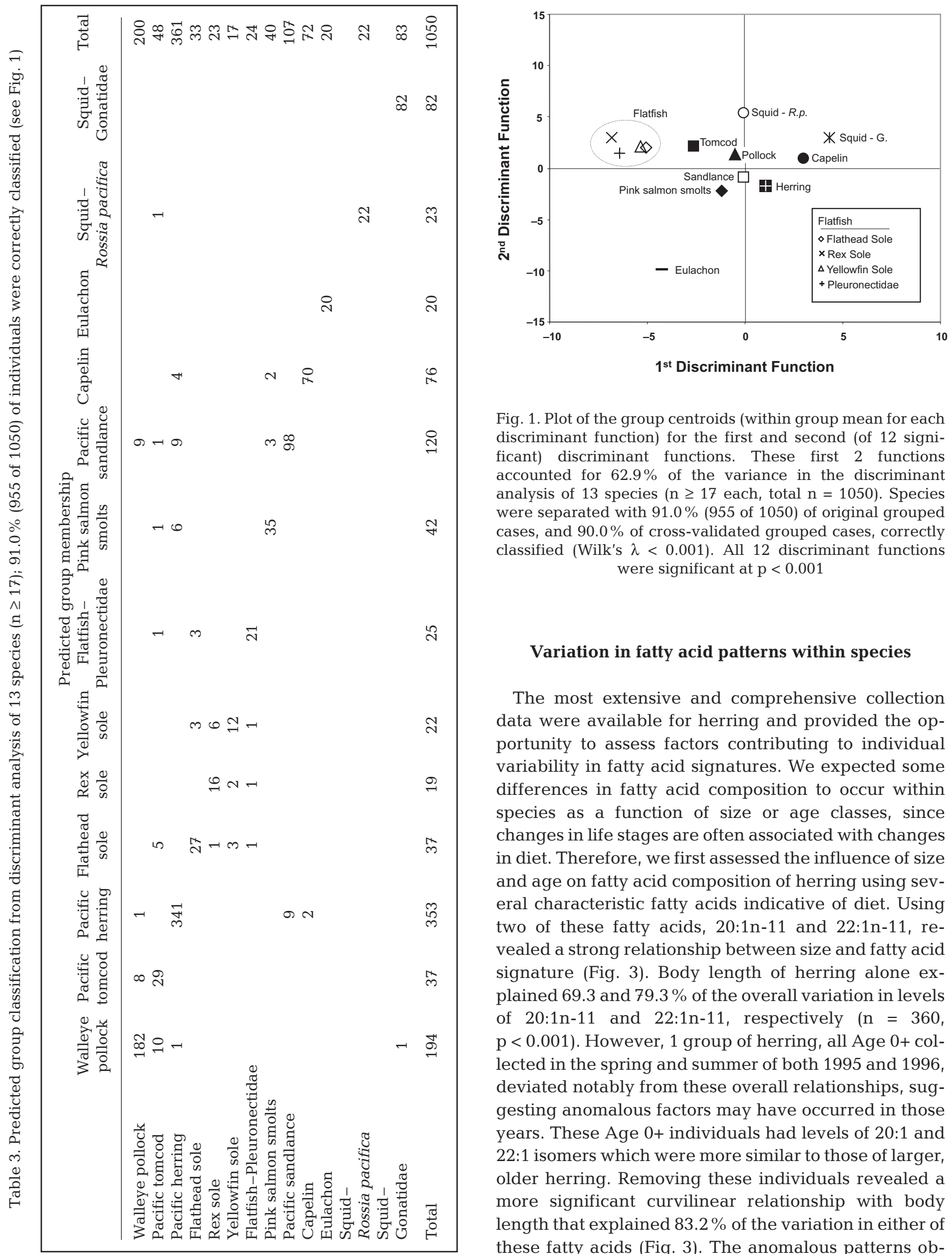

Fig. 1. Plot of the group centroids (within group mean for each discriminant function) for the first and second (of 12 significant) discriminant functions. These first 2 functions accounted for $62.9 \%$ of the variance in the discriminant analysis of 13 species $(n \geq 17$ each, total $n=1050)$. Species were separated with $91.0 \%$ (955 of 1050) of original grouped cases, and $90.0 \%$ of cross-validated grouped cases, correctly classified (Wilk's $\lambda<0.001$ ). All 12 discriminant functions were significant at $\mathrm{p}<0.001$

\section{Variation in fatty acid patterns within species}

The most extensive and comprehensive collection data were available for herring and provided the opportunity to assess factors contributing to individual variability in fatty acid signatures. We expected some differences in fatty acid composition to occur within species as a function of size or age classes, since changes in life stages are often associated with changes in diet. Therefore, we first assessed the influence of size and age on fatty acid composition of herring using several characteristic fatty acids indicative of diet. Using two of these fatty acids, $20: 1 \mathrm{n}-11$ and $22: 1 \mathrm{n}-11$, revealed a strong relationship between size and fatty acid signature (Fig. 3). Body length of herring alone explained 69.3 and $79.3 \%$ of the overall variation in levels of 20:1n-11 and 22:1n-11, respectively $(\mathrm{n}=360$, $\mathrm{p}<0.001$ ). However, 1 group of herring, all Age $0+$ collected in the spring and summer of both 1995 and 1996, deviated notably from these overall relationships, suggesting anomalous factors may have occurred in those years. These Age 0+ individuals had levels of 20:1 and 22:1 isomers which were more similar to those of larger, older herring. Removing these individuals revealed a more significant curvilinear relationship with body length that explained $83.2 \%$ of the variation in either of these fatty acids (Fig. 3). The anomalous patterns ob- 
served in Age 0+ herring from the spring and summer of 1995 and 1996 were also apparent in levels of other dietary indicator fatty acids, which otherwise exhibited a predictable relationship with size, accounting for 63 to $75 \%$ of their variance (Fig. 4). Two other species for which we had large sample sizes, juvenile pollock and sandlance $(11.5$ to $16.0 \mathrm{~cm})$, also had anomalously high levels of 20:1n-11, as well as of other isomers of 20:1 and 22:1, in 1995 and 1996 (Fig. 5).

Although the sampling of herring was not equally distributed across all regions, years and seasons, the sample size $(\mathrm{n}=360)$ was again sufficient to allow preliminary evaluation of such factors. We plotted the level of the fatty acid 20:1n-11 relative to body length to illustrate annual, seasonal and regional variation (Fig. 6a-c). Even though samples came from 4 yr, 3 seasons and 6 regions of PWS, the plots reveal that body length was the most important factor in explaining variation in levels of selected fatty acids (Fig. 6). Unfortunately, Age 0+ individuals from spring and summer 1995 and 1996 were the predominant herring sampled in NW and SW; no larger individuals were sampled in these areas. However, additional small herring were sampled in NW and SW in other years and followed the same patterns as those from other areas. Furthermore, 1995/1996 Age 0+ herring sampled in SC followed the same patterns as those in NW and SW, while larger SC herring followed the same patterns as in other areas (Fig. 6c). Thus, regardless of year, season or location, when 1995/1996 Age 0+ individuals were excluded, the relationships between body length and fatty acid levels were relatively consistent and explained most of the observed variation. Although, fatty acid levels in pollock and sandlance did not appear to be significantly affected by year (except 1995/1996 Age 0+ individuals from all locations), season or location, this could not be rigorously assessed beyond visual inspection. Because collections for most species were not equally distributed across sampling years, seasons and locations, the potential effects of these factors could not be assessed in other species.

Clear size-related differences in herring fatty acid signatures suggested that similar differences might occur in other species and would be useful in further characterizing species signatures. Thus, we conducted an additional discriminant analysis to examine age/

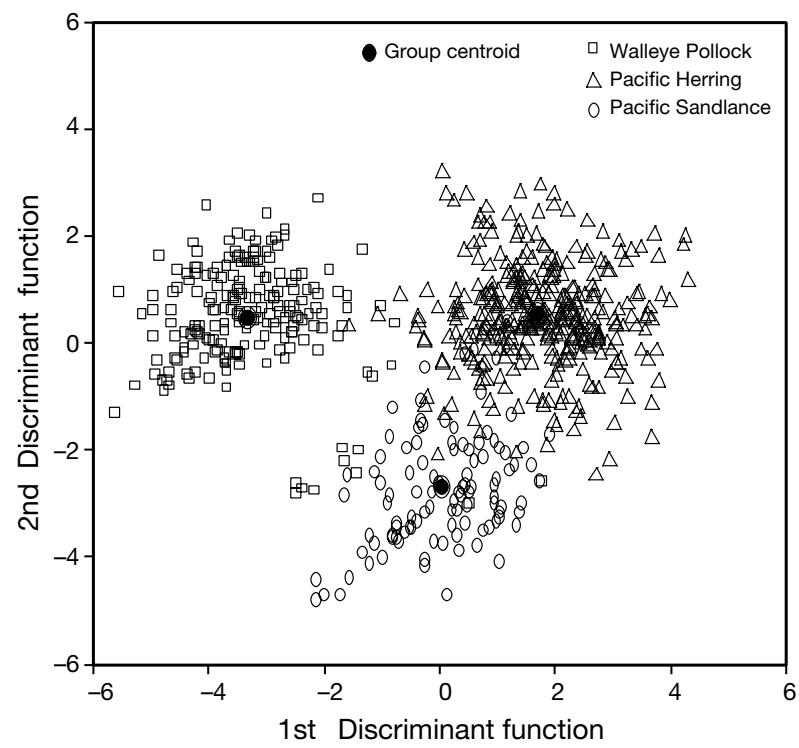

Fig. 2. Plot of the discriminant scores for the first and second discriminant functions for each individual as well as the group centroids from discriminant analysis performed on walleye pollock $(n=200)$, Pacific herring $(n=361)$ and Pacific sandlance $(n=107)$ alone. Both discriminant functions were significant $(\mathrm{p}<0.001)$, with the first discriminant function accounting for $77.8 \%$ of the variance; $95.1 \%$ of all original grouped cases and $94.5 \%$ of cross-validated grouped cases were correctly classified to species (Wilk's $\lambda<0.001$ ) 


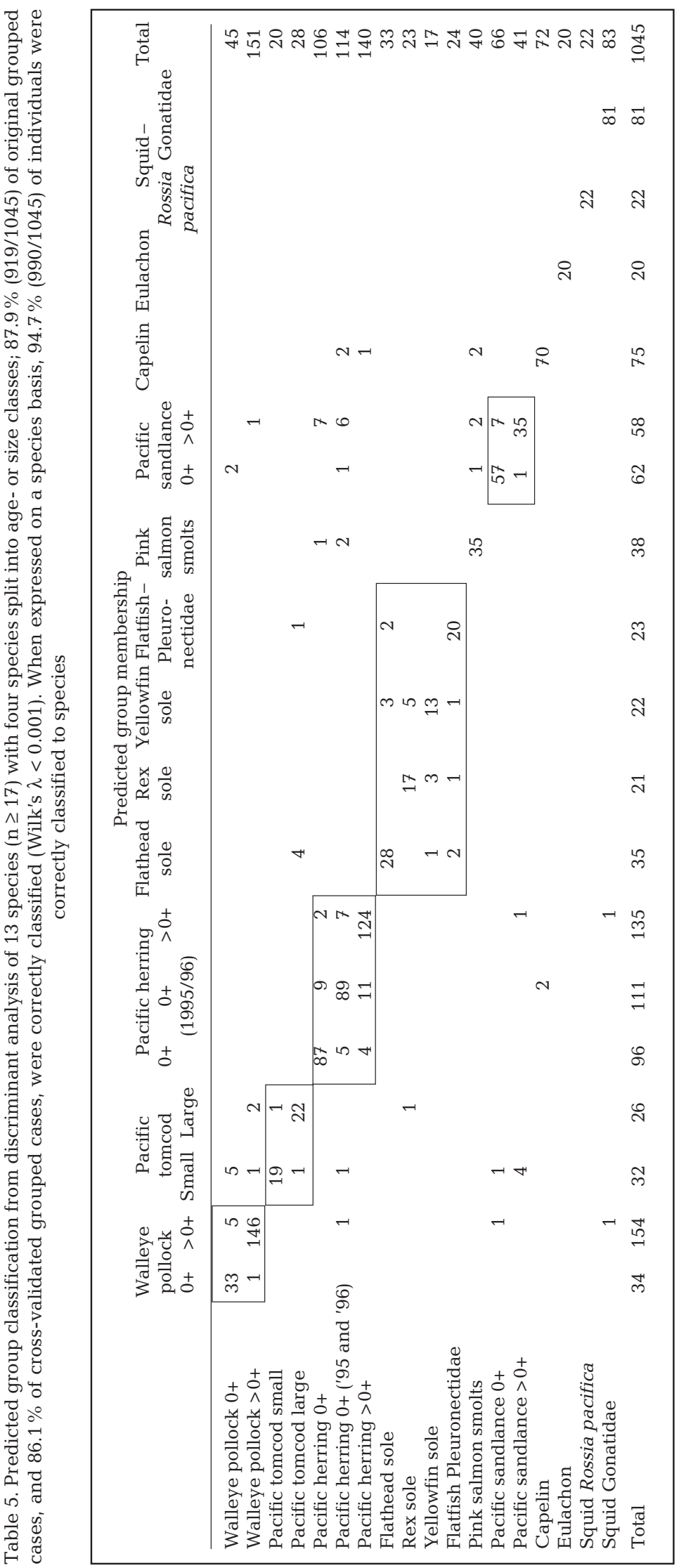

size-related differences for pollock, tomcod, herring and sandlance. We generally based our groupings on Age 0+ versus Age $>0+$ individuals, although in the case of tomcod we used $<$ or $>10.0 \mathrm{~cm}$ for division into small or large. Discriminant analysis of the original 13 species $(n \geq 17)$, but with pollock, tomcod, herring and sandlance divided into age or size classes, separated all groups with $87.9 \%$ of individuals correctly classified (Table 5). However, when classifications were expressed on a species basis, $94.7 \%$ of individuals were correctly classified to species. That is, misclassifications were predominantly among age groups within a species. Misclassifications of pollock as sandlance decreased (from 9 to 2 ) and the overall classifications of pollock and sandlance both improved from about 91 to $94 \%$ (Tables 3 \& 5). Small or large herring were most frequently misclassified as herring of other age classes and thus, the overall classification of herring remained at about $94 \%$. The classification of tomcod improved with separation into 2 size classes, with 43 of 48 (89.6\%) being correctly classified (Table 5), compared to 29 of 48 $(60.2 \%)$ in the first discriminant analysis (Table 3). Variation in fatty acid composition of other species, such as capelin, did not appear to have a predictable relationship with size or age, or were too few to split, however their classification rates were nearly identical in this analysis.

\section{DISCUSSION}

The results of our study demonstrate that most fish and invertebrate species in this ecosystem can be readily distinguished by their fatty acid signatures. In general, individuals were accurately classified to their species using both discriminant and CART analyses, raising the possibility of using fatty acid signatures to obtain quantitative estimates of species composition of predator diets (S. J. Iverson, C. Field, W. D. Bowen \& W. Blanchard unpubl. data, Iverson et al. 1999). However, it is clear that within-species variability is important to assess in order to characterize species patterns and evaluate influences on fat and fatty acid composition. Our results also indicate the potential use of fatty acid signatures to study finescale trophic interactions among fish species as well as shifts in the diets that may occur with 

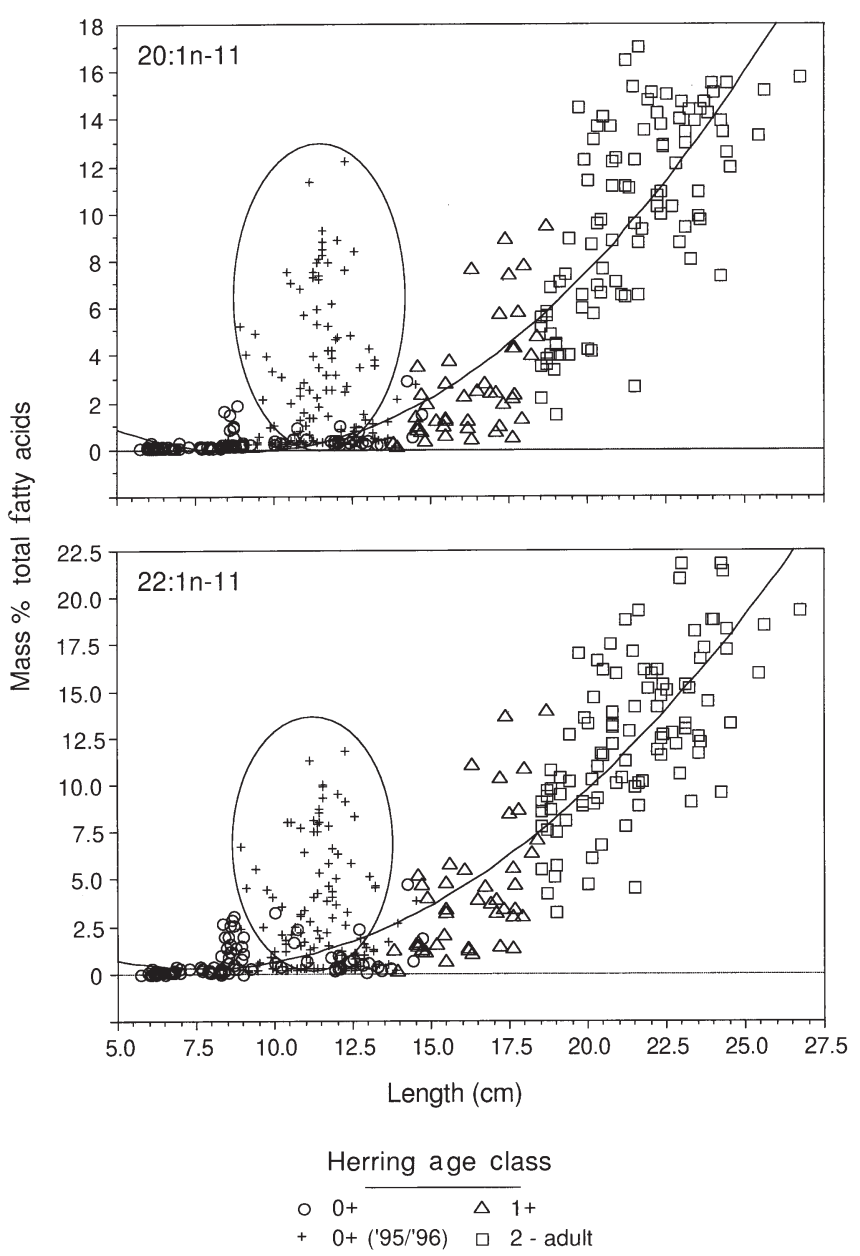

Fig. 3. Variation in 2 dietary indicator fatty acids in Pacific herring $(n=360)$ as a function of body length and age class. Age 0+ herring collected in the spring and summer of 1995 and 1996 (indicated within ovals) were excluded from illustrated regression equations (see 'Results'): 20:1n-11: $y=4.91-1.12 x+0.06 x^{2}$ $\left(\mathrm{r}^{2}=0.832, \mathrm{p}<0.001\right) ; 22: 1 \mathrm{n}-11: y=3.92-0.96 x+0.06 x^{2}$ $\left(\mathrm{r}^{2}=0.832, \mathrm{p}<0.001\right)$

factors such as body size, changes in primary and secondary production, or spatial/temporal differences in the physical environment.

PWS is a large estuarine system in the northern GOA that also has characteristics of a small inland sea (Niebauer et al. 1994). Although the overall understanding of forage fish populations throughout PWS is relatively fragmentary, in recent years a number of studies have been initiated to better understand distributions, abundance and resource partitioning among species (e.g. Duffy 1999). Our samples were collected in association with such studies and provide the opportunity to compare our results with other techniques in studies of the trophic ecology of these populations. Although we were not able to sample many species across years, seasons and locations, this study nevertheless represents the first comprehensive database of fat content and fatty acid composition of forage species in the northern GOA. These data are sufficient to permit preliminary assessment of the factors contributing to both species characteristics and individual variability in fatty acid signatures.
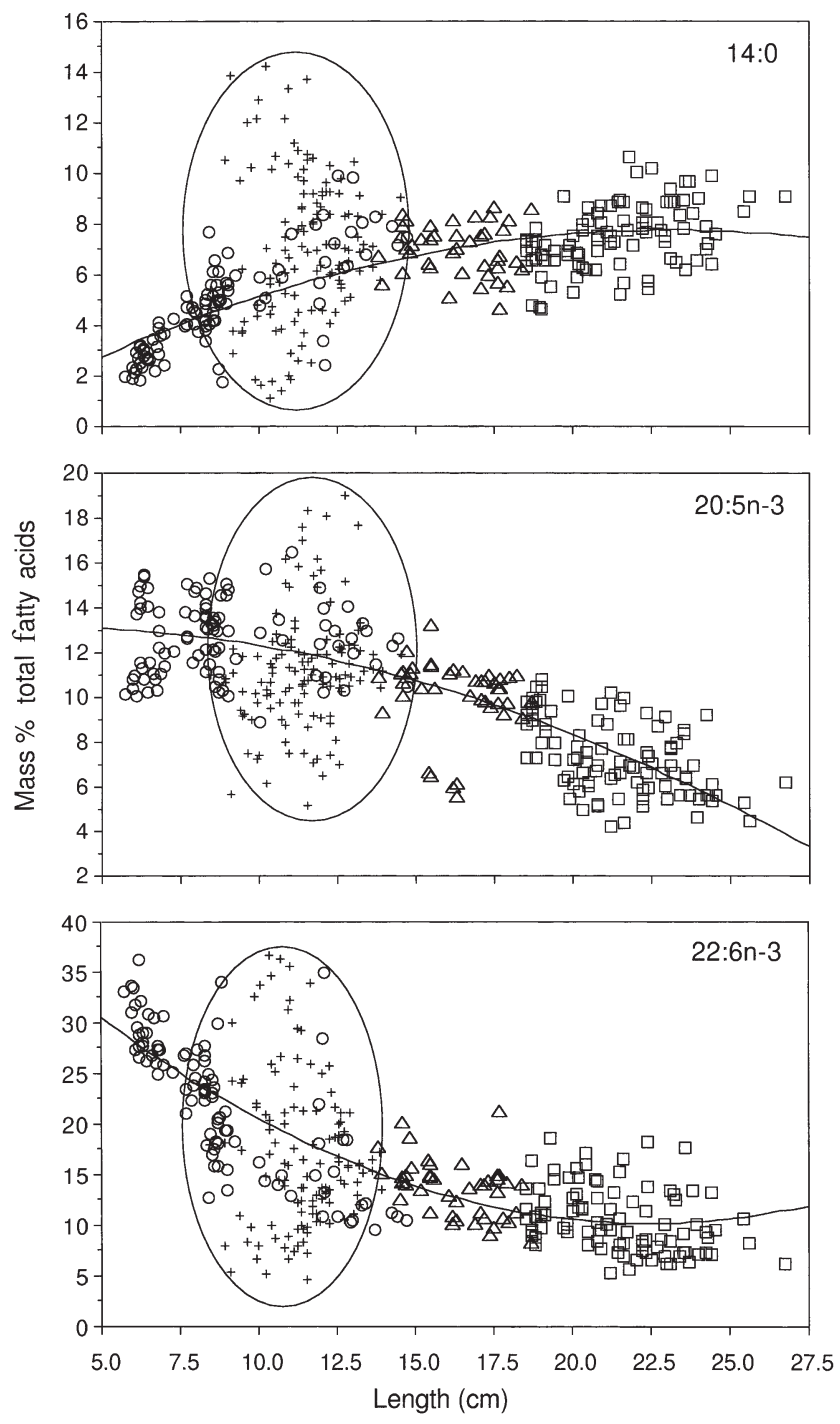

$$
\begin{aligned}
& \text { Herring age class } \\
& \circ 0+\frac{\triangle 1+}{+} 0+(' 95 / 96) \quad \square 2 \text { - adult }
\end{aligned}
$$

Fig. 4. Variation in 3 dietary indicator fatty acids in Pacific herring $(n=360)$ as a function of body length and age class. Age $0+$ herring collected in the spring and summer of 1995 and 1996 (indicated within ovals) were excluded from illustrated regression equations (see 'Results'): 14:0: $y=-0.37+0.70 x-0.02 x^{2}$ $\left(\mathrm{r}^{2}=0.629, \mathrm{p}<0.001\right) ; 20: 5 \mathrm{n}-3: y=13.07+0.08 x-0.02 x^{2}$ $\left(\mathrm{r}^{2}=0.691, \mathrm{p}<0.001\right) ; 22: 6 \mathrm{n}-3: y=43.86-3.01 x+0.07 x^{2}$ $\left(\mathrm{r}^{2}=0.751, \mathrm{p}<0.001\right)$ 


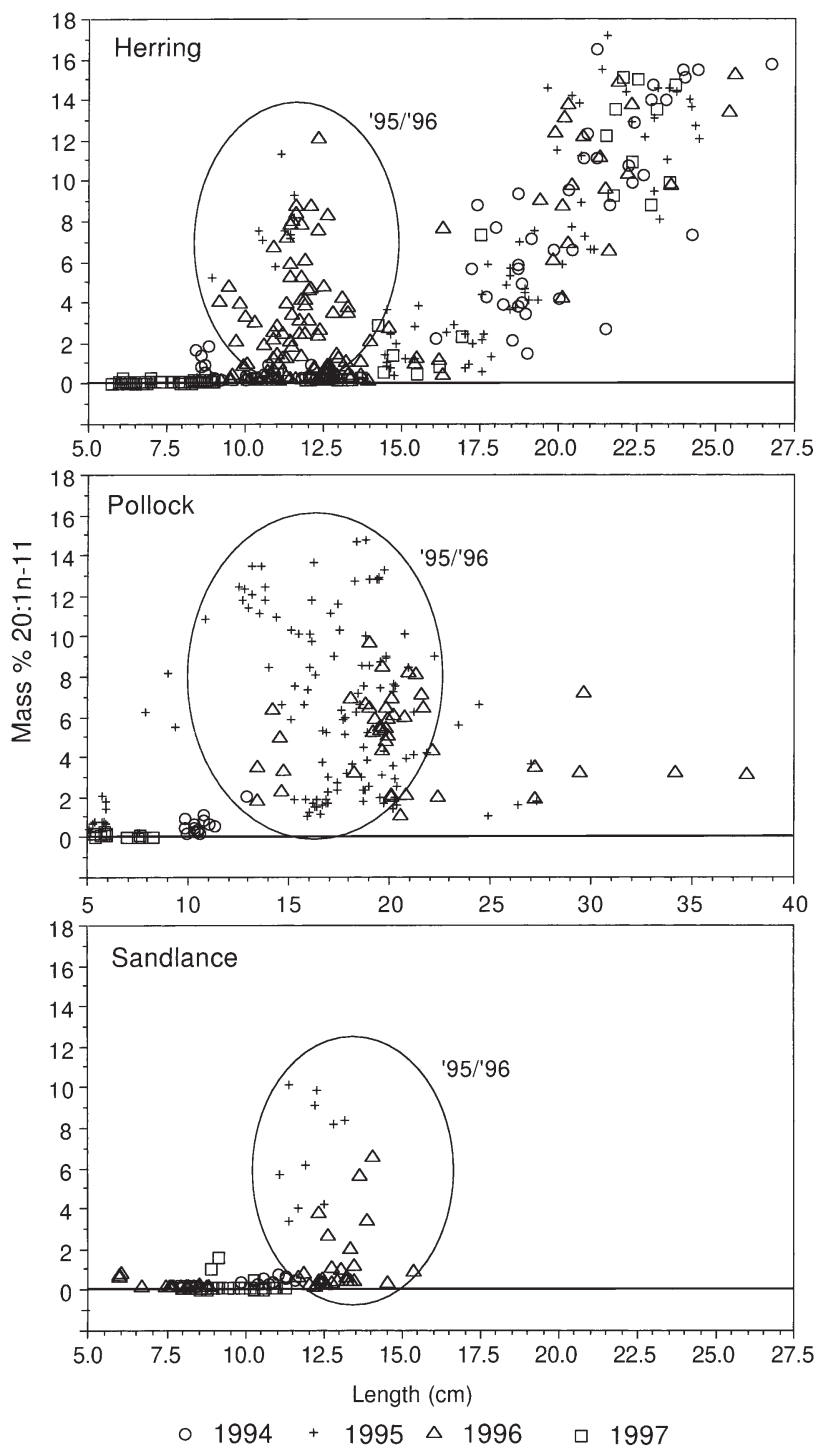

Fig. 5. Variation in 1 indicator fatty acid 20:1n-11 in Pacific herring $(n=360)$ compared with walleye pollock $(n=196)$ and Pacific sandlance $(n=107)$ as a function of body length and illustrating distribution of points across years. Points contained within ovals indicate size classes of herring, pollock and sandlance averaging 11.5, 16.0 and $12.5 \mathrm{~cm}$, respectively, collected in 1995 and 1996

Fat content varied greatly both between and sometimes within species, usually associated with season. Consistent with previous studies (e.g. Anthony \& Roby 1997), eulachon were uniformly highest in fat content at 15.0 to $25.3 \%$, making them important potential prey for piscivorous seabirds, pinnipeds and cetaceans (Pitcher 1977, Seaman et al. 1982, Calkins 1983, Vermeer 1992). Pacific herring were overall the next highest in fat content, but this varied widely and was lowest in spring and highest in older individuals in summer and fall (Table 1), which is consistent with other studies that have measured high fat contents in pre-spawning herring (Funk \& Sandone 1990, Anthony \& Roby 1997). (Note: our fat content results are not comparable with those of Payne et al. 1999, as they used the Bligh
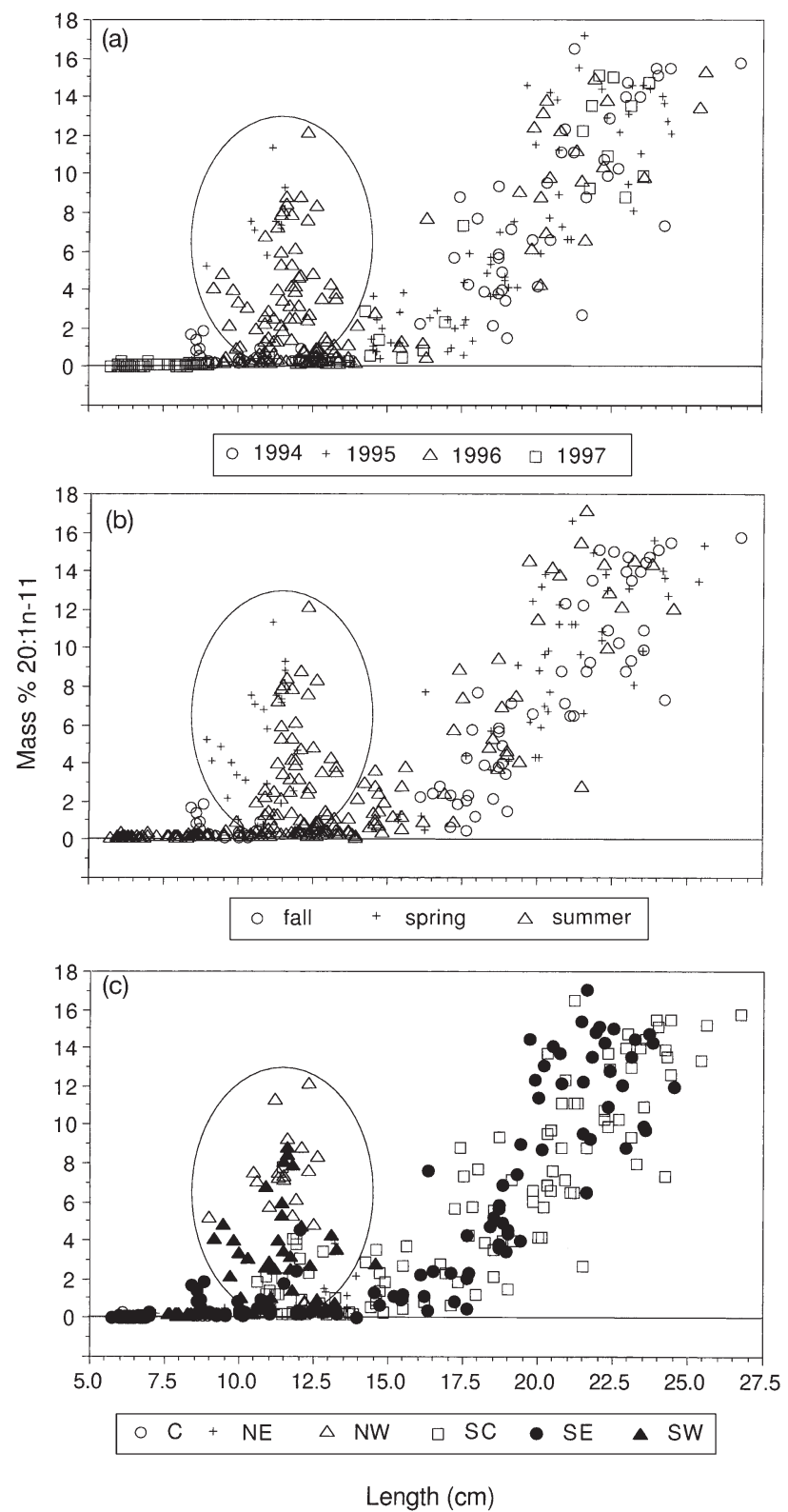

Fig. 6. Variation in 1 indicator fatty acid 20:1n-11 in Pacific herring $(n=360)$ as a function of body length and illustrating distribution of points (a) across years, (b) across seasons and (c) across locations within PWS, Alaska. Individuals of 0+ age class collected in the spring and summer of 1995 and 1996 (within ovals), which were excluded from the illustrated regression analysis (see Fig. 3): body length alone explained $83.2 \%$ of the variation in this fatty acid regardless of year, season or location 
\& Dyer method for lipid extraction which can significantly underestimate total fat content; see 'Methods', Iverson et al. 2001a). The highest fat contents of both capelin $(3.2 \%$, spring) and sandlance $(7.8 \%$, summer $)$ were also consistent with measurement prior to spawning (i.e. late spring-early summer in capelin, late summer or fall in sandlance; (Montevechhi \& Piatt 1984, Anthony \& Roby 1997, Robards et al. 1999). In contrast, pollock may spawn intermittently year round (Fritz et al. 1993) and hence, seasonal associations with variable, but usually relatively low, fat contents are less clear. The species with the third highest fat content was the squid Berrytheuthis magister at up to $13.2 \%$ fat (Table 1). Although squid are generally thought to be low in fat (e.g. $\leq 2 \%$ in Illex illecebrosus, Kirsch et al. 1998), our B. magister values are consistent with similarly high values measured in $B$. magister in the Bering Sea (M. E. Goebel \& S. J. Iverson unpubl. data) and in the deep-sea squid Gonatus fabricii from the Norwegian Sea (Hooker et al. 2001), and indicate that this notion needs to be reconsidered for some species. The consistently lowest and least variable fat contents were generally found among the flatfish species, shrimps and octopus. Thus, in general, despite some degree of seasonality, greatly differing fat and energy contents tend to distinguish different species of fish and invertebrates.

Fatty acid signatures generally characterized forage species with considerable accuracy. As stated earlier, we did not remove stomach contents of individuals as we wished to characterize them as prey which are eaten whole by marine predators. This may have resulted in increased variability within species and hence a more conservative classification success. Nevertheless, up to $95 \%$ of individuals $(n=1143)$ from 22 species were correctly classified using either CART or discriminant analyses (Tables 4 \& 5). However, there was also evidence of some overlap in signatures of certain species. Marine fish and invertebrate fatty acid signatures will be affected mainly by diet, as well as to some degree by the biosynthesis of certain fatty acids. Diets within and among fish and invertebrate species will in turn reflect characteristics of the prey field as well as morphological constraints and life style of the predators. Additionally, although overall diets will differ among species, there may be some general overlap, and perhaps considerable short-term overlap in particular regions or seasons when certain shared prey are abundant. Our discriminant plots illustrate spatial relationships between groups and demonstrate that species with similar lifestyles and diets tended to cluster closer together, whereas those with the greatest differences in life history characteristics differed most in fatty acid patterns. Hence, it is useful to compare these findings with those of previous analyses using tools such as stomach contents analysis. For instance, within the finfish, the flatfish species, which have all been documented to feed primarily on benthic infauna and epifauna, were more similar to one another and differed most from the more pelagic zooplanktivores and piscivores such as herring and pollock (e.g. Fig. 2). Eulachon differed most from all other finfish, cephalopod or crustacean species studied. The unique fatty acid signature of eulachon was characterized by extremely high levels of 18:1n-9, moderately high levels of $14: 0$ and $16: 1 \mathrm{n}-7$, and extremely low levels of polyunsaturated fatty acids such as 20:5n-3 and 22:6n-3 (Table 2). Although eulachon spawn and hatch in fresh water (Gillespie \& Westrheim 1997), once at sea little is known of their distribution and diets (Hay et al. 1997). Thus, the source of their unique fatty acid patterns observed is presently unclear.

Three additional species (pollock, herring and sandlance) showed some degree of overlap in fatty acid signatures and although generally well-separated (at 91 to $96 \%$ ), were thus occasionally misclassified as one another (e.g. Figs. 1 \& 2, Table 3). In general, stomach contents analysis has shown diets of larval walleye pollock to consist of copepod eggs and nauplii; as larvae grow they begin to feed on larger prey such as small copepods. As adults, pollock feed both in mid-water and demersally on a wide variety of copepods, euphasiids and fish (primarily juvenile pollock but also other species) and as adult pollock grow they tend to take increasingly larger food items (Incze et al. 1988, NRC 1996). Likewise, Pacific herring consume plankton (copepod larvae) as juveniles, switching to both larger plankton and fish as they mature (Grosse \& Hay 1988). Pacific sandlance feed on copepod eggs and nauplii as larvae and on a variety of zooplankton (especially calanoid copepods) and crustaceans as they mature (Field 1988). Most data also suggest sandlance feed between mid-water and the surface, not at the bottom. Hence, these 3 species share broad overlap in diets during several life stages. In PWS, juvenile pollock and juvenile herring are sometimes found in mixed schools, and stomach content analyses of pollock, herring and sandlance indicate a significant degree of dietary overlap, especially between pollock and herring collected in the same locations (Sturdevant 1996). Some dietary overlap has also been reported between sandlance and capelin and between pink and chum salmon in PWS (Willette et al. 1997). Thus, dietary overlap likely accounts for the proximity of these species on discriminant plots and the resulting misclassifications of some individuals (Tables $3 \& 5$ ), despite very good overall classification rates (Tables 4 \& 5).

For a number of fish species, especially those with a large range in body size, diets change with increasing size as described above for herring and pollock. Larger 
fish with concurrent changes in mouth morphology can consume larger or different prey (Keast \& Webb 1966, McEachran et al. 1976, Ross 1977, Hacunda 1981), which results in fatty acid differences within species (Logan et al. 2000). In our herring samples, body size was the most significant factor in explaining variation in fatty acid signature (Figs. 3 \& 6), which is consistent with known changes in herring diets with size (Grosse \& Hay 1988). Additionally, when other species such as pollock and tomcod were separated into size or age classes, their overall species classification was usually improved, averaging $94.7 \%$ (Table 5).

PWS is a complex embayment with differing fresh and salt water inputs, which might be expected to affect fatty acid signatures due to differences in regional prey abundance or in primary and secondary production. However, from our data, there was no clear evidence for a significant influence of location within PWS on the fatty acid signatures of herring (e.g. Fig. 6). Nevertheless, this remains to be more rigorously tested in both this and other species.

Finally, studies using stomach contents analysis have shown that fish diets can exhibit considerable individual, seasonal or interannual variation, and this appears to be reflected in their fatty acid signatures. For instance, during the spring bloom in the northern GOA, the later life stages of both walleye pollock and Pacific herring are found in the ocean surface layers where they feed heavily on large calanoid copepods, although both are also piscivorous. Planktivory usually predominates diets during the peak bloom but this switches to nekton (fish and squid) and a variety of other prey as the bloom declines (Foy \& Norcross 1999, Willette et al. 1999). During 1995 and 1996, there is evidence that physical oceanography in PWS differed (Kline 2000) and that the spring ocean surface layer was more highly stratified than normal and was associated with higher salinities at depth (Willette et al. 1999). During this period, Willette et al. (1999) found that both juvenile pollock and herring switched early to diets of predominantly nekton prey, coincident with an early spring decline in biomass of calanoid copepods. Whether these diet shifts were due to changes in overall prey availability or changes in spatial distribution and feeding habits of juvenile pollock and herring is not known. However, fatty acid signatures of the 1995/1996 spring and summer samples in our study were consistent with this early switch to nekton prey and deviated from the patterns in fatty acid signatures that were otherwise mostly explained by body size alone (Figs. 3, 4 \& 6). This was particularly evident for Age $0+$ herring. High levels of fatty acids $20: 1 n-11$ and 22:1n-11, which generally characterized older herring, were found in juvenile herring in 1995 and 1996, but not in other years (Figs. $3 \& 6$ ). Because the overall fatty acid signatures of these juveniles were simply similar to those of adults, all herring were nevertheless well classified (94 to $98 \%$ ) from other species. Elevated levels of these fatty acids were also apparent in similar size classes of pollock and sandlance during these years (Fig. 5), suggesting perhaps similar dietary shifts in these 2 species. However, again, pollock and sandlance individuals remained well classified (91 to $95 \%$ ) from other species overall. Since changes in the diet of a fish can result in a significant change in its fatty acid signature in less than $3 \mathrm{wk}$ (Kirsch et al. 1998), our findings as well as those of others (e.g. St. John \& Lund 1996, Saito \& Murata 1998, Mayzaud et al. 1999) clearly indicate that fatty acids can be used to detect dietary changes. The ability to detect such changes should prove valuable in studying trophic interactions and further understanding complex marine food webs.

In summary, our results demonstrate that fatty acid signature analyses, combined with the use of other techniques, can be a valuable tool in providing insight into ecological relationships at a number of trophic levels. Despite individual variation within species, fatty acid signatures provide a powerful tool for identifying fish and invertebrate species. The 22 species analyzed were distinguished with $95 \%$ accuracy overall. For herring (the species for which we had the largest sample size), size class had the strongest effect on fatty acid signature, although interannual shifts in the diets of 1 age class were also detected. Nevertheless, even with the variation observed among individuals, herring as a whole were 95 to $98 \%$ correctly distinguished from all other species (Tables 4 \& 5). Quite remarkably, pollock over a size range of 5 to $38 \mathrm{~cm}$ and 1 to $367 \mathrm{~g}$ were distinguished from other species with 91 to $95 \%$ accuracy. These results demonstrate that betweenspecies variability is usually much greater than within species variability. However, our results also indicate that some species will be more difficult to distinguish from one another and will require careful evaluation. Our findings have important implications for evaluating diets and food web interactions at higher trophic levels. If fatty acid signatures can accurately characterize fish and other prey species, they can then be used to study and perhaps estimate diets of predators of these species (i.e. Iverson et al. 1999, S. J. Iverson, C. Field, W. D. Bowen \& W. Blanchard unpubl. data). Additionally, the potential for evaluating size classes of some prey species eaten by higher level predators also exists and may provide more accuracy in distinguishing similar species.

Acknowledgements. We thank C. Beck, A. Bowen, J. Lassner, M. Logan, J. Marcus and L. Smith for laboratory assistance, and personnel associated with the SEA and APEX projects of the Exxon Valdez Restoration Science Studies Program for 
their assistance in providing samples of fish for this study. We also thank E. Brown and T. Kline for useful discussions and W. D. Bowen for helpful comments on an earlier version of the manuscript. This study was conducted as part of the Exxon Valdez Oil Spill Restoration Program, funded by the Exxon Valdez Oil Spill Trustee Council. Additional support was provided by Natural Sciences and Engineering Research Council (NSERC, Canada) research and equipment grants to S.J.I.

\section{LITERATURE CITED}

Ackman RG (1980) Fish lipids. Part 1. In: Connell JJ (ed) Advances in fish science and technology. Fishing News Books, Farnham, p 86-103

Ackman RG (1991) Application of gas-liquid chromatography to lipid separation and analysis: qualitative and quantitative analysis. In: Perkins EG (ed) Analyses of fats, oils and lipoproteins. Amer Oil Chem Soc, Champagne, p 270-300

Ackman RG, Eaton CA (1966) Lipids of the fin whale (Balaenoptera physalus) from North Atlantic waters. III. Occurence of eicosenoic and docosenoic fatty acids in the zooplankton Meganyctiphanes norvegica (M. Sars) and their effect on whale oil composition. Can J Biochem 44: 1561-1566

Ackman RG, Ratnayake WMN, Olsson B (1988) The 'basic' fatty acid composition of Atlantic fish oils: potential similarities useful for enrichment of polyunsaturated fatty acids by urea complexation. J Am Oil Chem Soc 65: $136-138$

Anthony JA, Roby DD (1997) Variation in lipid content of forage fishes and its effect on energy provisioning rates to seabird nestlings. In: Forage fishes in marine ecosystems. Proc Int Symp Role of Forage Fishes in Marine Ecosystems. University of Alaska Sea Grant College Program, Report No. 97-01, p 725-729

Bailey SK, Well DE (1994) The measurement of lipids as a cofactor for organic contaminants in biota. Proceedings of the QUASIMEME Lipid Workshop, Dublin

Blackburn JE, Anderson PJ (1997) Pacific Sand Lance growth, seasonal availability, movements, catch variability, and food in the Kodiak-Cook Inlet area of Alaska. In: Forage fishes in marine ecosystems. Proc Int Symp Role of Forage Fishes in Marine Ecosystems. University of Alaska Sea Grant College Program, Report No. 97-01, p 409-426

Bligh EG, Dyer WJ (1959) A rapid method of total lipid extraction and purification. Can J Biochem Physiol 37:911-917

Bowen WD, Siniff DB (1999) Distribution, population biology, and feeding ecology of marine mammals. In: Reynolds JE, Rommel SA (eds) Biology of marine mammals. Smithsonian Institution Press, Washington, DC, p 423-484

Calkins DG (1983) Susitna hydroelectric project phase II 1982 annual report: big game studies. Vol IX, Belukha whale. Alaska Department of Fish \& Game, $15 \mathrm{p}$

Christie WW (1982) Lipid analysis. Pergamon Press, New York

Ciannelli L (1997) Winter dormancy in the Pacific Sand Lance (Ammodytes hexapterus) in relation to gut evacuation time. In: Forage fishes in marine ecosystems. Proc Int Symp Role of Forage Fishes in Marine Ecosystems. University of Alaska Sea Grant College Program, Report No. 97-01, p 95-104

Clark LA, Pregibon D (1992) Tree-based models. In: Chambers JM, Hastie TJ (eds) Statistical models. S. Wadsworth \& Brooks/Cole Advanced Books \& Software, Pacific Grove, CA, p 377-419

Cook HW (1985) Fatty acid desaturation and chain elongation in eucaryotes. In: Vance DE, Vance JE (eds) Biochemistry of lipids and membranes. Benjamin/Cummings, Menlo Park, CA, p 181-211

Duffy DC (1999) APEX project: Alaska predator ecosystem experiment in Prince William Sound and the Gulf of Alaska. Exxon Valdez Oil Spill Restoration Project annual report, No. 98163 A-T. Paumanok Solutions, Kailua, HI

Field LJ (1988) Pacific Sand Lance, Ammodytes hexapterus, with notes on related Ammodytes species. In: Wilimovsky NJ, Incze LS, Westerheim SJ (eds) Species synopses: life histories of selected fish and shellfish of the northeast Pacific and Bering Sea. Washington Sea Grant Program and Fisheries Research Institute, Seattle, WA, p 23-33

Folch J, Lees M, Sloane-Stanley GH (1957) A simple method for the isolation and purification of total lipids from animal tissues. J Biol Chem 226:497-509

Foy RJ, Norcross BL (1999) Spatial and temporal variability in the diet of juvenile Pacific herring (Clupea pallasi) in Prince William Sound, Alaska. Can J Zool 77:697-706

Fraser AJ, Sargent JR, Gamble JC, Seaton DD (1989) Formation and transfer of fatty acids in an enclosed marine food chain comprising phytoplankton, zooplankton and herring (Clupea harengus L.) larvae. Mar Chem 27:1-18

Fritz LW, Wespestad VG, Collie JS (1993) Distribution and abundance trends of forage fishes in the Bering Sea and Gulf of Alaska. In: (Anon) Is it food? Addressing marine mammal and seabird declines. Workshop summary. Alaska Sea Grant, No. 93-01. University of Alaska Fairbanks, AK, p 30-44

Frost KJ, Lowry LF (1994) Assessment of injury to harbor seal in Prince William Sound, Alaska, and adjacent areas following the Exxon Valdez oil spill. Final report, marine mammal study No. 5. State Federal Resource Damage Assessment, $154 \mathrm{p}$

Frost KJ, Lowry LF, VerHoef JM (1999) Monitoring the trend of harbor seals in Prince William Sound, Alaska, after the Exxon Valdez oil spill. Mar Mamm Sci 15:494-506

Funk FC, Sandone GJ (1990) Catch-age analysis of Prince William Sound, Alaska, herring 1973-1988. Fish Res Bull No. 90-01, Alaska Department of Fish \& Game, Juneau

Gillespie GE, Westrheim SJ (1997) Synopsis of information on marine fishes utilized as prey by marine and shoreline birds of the Queen Charlotte Islands. In: Vermeer K, Morgan KH (eds) The ecology, status and conservation of marine birds of the Queen Charlotte Islands. Can Wildl Serv Occas Pap 93:36-55

Graeve M, Hagen W, Kattner G (1994a) Herbivorous or omnivorous? On the significance of lipid compositions as trophic markers in Antarctic copepods. Deep-Sea Res Part I 41:915-924

Graeve M, Kattner G, Hagen W (1994b) Diet-induced changes in the fatty acid composition of Arctic herbivorous copepods: experimental evidence of trophic markers. J Exp Mar Biol Ecol 182:97-110

Grosse DJ, Hay DE (1988) Pacific Herring, Clupea harengus pallasi, in the Northeast Pacific and Bering Sea. In: Wilimovsky NJ, Incze LS, Westerheim SJ (eds) Species synopses: life histories of selected fish and shellfish of the northeast Pacific and Bering Sea. Washington Sea Grant Program and Fisheries Research Institute, Seattle, WA, p 34-54

Hacunda JS (1981) Trophic relationships among demersal fishes in a coastal area of the Gulf of Maine. Fish Bull 79: 775-788

Hay DE, Boutillier J, Joyce M, Langford G (1997) The eulachon (Thaleichthys pacificus) as an indicator species in the North Pacific. In: Proc Int Symp Role of Forage Fishes in 
Marine Ecosystems. University of Alaska Sea Grant College Program, Report No. 97-01, p 509-530

Hayes DL, Kuletz KJ (1997) Decline of Pigeon Guillemot populations in Prince William Sound, Alaska, and apparent changes in distribution and abundance of their prey. In: Forage fishes in marine ecosystems. Proc Int Symp Role of Forage Fishes in Marine Ecosystems. University of Alaska Sea Grant College Program, Report No. 97-01, p 699-702

Hooker SK, Iverson SJ, Ostrum P, Smith SC (2001) Diet of northern bottlenose whales inferred from fatty-acid and stable-isotope analyses of biopsy samples. Can J Zool 79: 1442-1454

Hooper SN, Paradis M, Ackman RG (1973) Distribution of trans-6-hexadecanoic acid, 7-methyl-7-hexadecanoic acid and common fatty acids in lipids of the ocean sunfish Mola mola. Lipids 8:509-516

Incze LS, Lynde CM, Kim S, Strickland R (1988) Walleye Pollock, Theragra chalcogramma, in the eastern Bering Sea. In: Wilimovsky NJ, Incze LS, Westerheim SJ (eds) Species synopses: life histories of selected fish and shellfish of the northeast Pacific and Bering Sea. Washington Sea Grant Program and Fisheries Research Institute, Seattle, WA, p 57-69

Iverson SJ (1993) Milk secretion in marine mammals in relation to foraging: can milk fatty acids predict diet? Symp Zool Soc Lond 66:263-291

Iverson SJ, Oftedal OT, Bowen WD, Boness DJ, Sampugna J (1995) Prenatal and postnatal transfer of fatty acids from mother to pup in the hooded seal. J Comp Physiol 165:1-12

Iverson SJ, Arnould JPY, Boyd IL (1997a) Milk fatty acid signatures indicate both major and minor shifts in the diet of lactating Antarctic fur seals. Can J Zool 75:188-197

Iverson SJ, Frost KJ, Lowry LF (1997b) Fatty acid signatures reveal fine scale structure of forgaing distribution of harbor seals and their prey in Prince William Sound, Alaska. Mar Ecol Prog Ser 151:255-271

Iverson SJ, Frost KJ, Lang S (1999) The use of fatty acid signatures to investigate foraging ecology and food webs in Prince William Sound, Alaska: harbor seals and their prey. In: Monitoring, habitat use, and trophic interactions of harbor seals in Prince William Sound, Alaska. Exxon Valdez Oil Spill Trustee Council annual report, No. 97064. Alaska Department of Fish and Game, Division of Wildlife Conservation, Fairbanks, AK, p 38-102

Iverson SJ, Lang SLC, Cooper MH (2001a) Comparison of the Bligh and Dyer and Folch methods for total lipid determination in a broad range of marine tissue. Lipids 36: 1283-1287

Iverson SJ, McDonald JE, Smith LH (2001b) Changes in diet of free-ranging black bears in years of contrasting food availability revealed through milk fatty acids. Can J Zool 79:2268-2279

Jobling M, Breiby A (1986) The use and abuse of fish otoliths in studies of feeding habits of marine piscivores. Sarsia 71: 265-274

Keast A, Webb D (1966) Mouth and body form relative to feeding ecology in the fish fauna of a small lake, Lake Opinicon, Ontario. J Fish Res Board Can 23:1845-1873

Kharlamenko VI, Zhukova NV, Khotimchenko SV, Svetachev VI, Kamenev GM (1995) Fatty acids as markers of food sources in a shallow-water hydrothermal ecosystem (Kraternaya Bight, Yankich Island, Kurile Islands). Mar Ecol Prog Ser 120:231-241

Kirsch PE, Iverson SJ, Bowen WD, Kerr SR, Ackman RG (1998) Dietary effects on the fatty acid signature of whole Atlantic cod (Gadus morhua). Can J Fish Aquat Sci 55: 1378-1386
Kirsch PE, Iverson SJ, Bowen WD (2000) Effect of a low-fat diet on body composition and blubber fatty acids of captive juvenile harp seals (Phoca groenlandica). Physiol Biochem Zool 73:45-59

Klem A (1935) Studies in the biochemistry of whale oils. Hvalradets Skr 11:49-108

Kline TC Jr (2000) Evidence of biophysical coupling from shifts in natural stable carbon and nitrogen isotopes in Prince William Sound, Alaska. Wakefield Symp Spatial Processes and Management of Marine Populations. University of Alaska Sea Grant, Fairbanks, AK, Report No. AK-00-04

Klungsøyr J, Tilseth S, Wilhelmsen S, Falk-Petersen S, Sargent JR (1989) Fatty acid composition as an indicator of food intake in cod larvae Gadus morhua from Lofoten, Northern Norway. Mar Biol 102:183-188

Lee RF, Nevenzel JC, Paffenhofer GA (1971) Importance of wax esters and other lipids in the marine food chain: phytoplankton and copepods. Mar Biol 9:99-108

Legendre P, Legendre L (1998) Numerical ecology. 2nd English edn. Elsevier Scientific Publishers, Amsterdam

Logan MS, Iverson SJ, Ruzzante DE, Walde SJ, Machi PJ, Alonso MF, Cussac VE (2000) Long term diet differences between morphs in trophically polymorphic Percichthys trucha populations from the southern Andes. Biol J Linn Soc 69:599-616

Mayzaud P, Virtue P, Albessard E (1999) Seasonal variations in the lipid and fatty acid composition of the euphausiid Megancyctiphanes norvegica from the Ligurian Sea. Mar Ecol Prog Ser 186:199-210

McEachran JD, Boesch DF, Musick JA (1976) Food division within two sympatric species-pairs of skates (Pisces: Rajidae). Mar Biol 35:301-317

Merrick RL, Chumbley MK, Byrd GV (1997) Diet diversity of Steller sea lions (Eumetopias jubatus) and their population decline in Alaska: a potential relationship. Can J Fish Aquat Sci 54:1342-1348

Montevechhi WA, Piatt J (1984) Composition and energy content of mature inshore spawning capelin (Mallotus villosus): implications for seabird predators. Comp Biochem Physiol 78:15-20

Niebauer HJ, Royer TC, Weingartner TJ (1994) Cirulation of Prince William Sound, Alaska. J Geophys Res 99:1411314126

NRC (National Research Council) (1996) The Bering Sea ecosystem. Committee on the Bering Sea. National Academic Press, Washington, DC

Payne SA, Johnson BA, Otto RS (1999) Proximate composition of some north-eastern Pacific forage fish species. Fish Oceanogr 8(3):159-177

Pierce GJ, Boyle PR (1991) A review of methods for diet analysis in piscivorous marine mammals. Oceanogr Mar Biol Annu Rev 29:409-486

Pitcher KW (1977) Population productivity and food habits of harbor seals in the Prince William Sound-Copper River Delta area, Alaska. US Marine Mammal Commission Final Report, No. MMC-75/03, $36 \mathrm{p}$

Popova OA (1978) The role of predaceous fish in ecosystems. In: Gerking SD (ed) Ecology of freshwater fish production. Blackwell Scientific Publishers, London, p 215-249

Raclot T, Groscolas R, Cherel Y (1998) Fatty acid evidence for the importance of myctophid fishes in the diet of king penguins, Aptenodytes patagonicus. Mar Biol 132:523-533

Robards MD, Piatt JF, Rose GA (1999) Maturation, fecundity, and intertidal spawning of Pacific sandlance (Ammodytes hexapterus) in the northern Gulf of Alaska. J Fish Biol 54: 1050-1068 
Ross ST (1977) Patterns of resource partitioning in searobins (Pisces: Triglidae). Copeia 3:561-571

Rouvinen K, Kiiskinen T (1989) Influence of dietary fat source on the body fat composition of mink (Mustela vison) and blue fox (Alopex lagopus). Acta Agric Scand 39:279-288

Saito H, Murata M (1998) Origin of the monoene fats in the lipid of midwater fishes: relationship between the lipids of myctophids and those of their prey. Mar Ecol Prog Ser 168: 21-33

Sargent JR, Parkes RJ, Mueller-Harvey I, Henderson RJ (1988) Lipid biomarkers in marine ecology. In: Sleigh MA (ed) Microbes in the sea. Ellis Horwood, Chichester, p 119-138

Seaman GA, Lowry LF, Frost KJ (1982) Foods of belukha whales (Delphinapterus leucas) in western Alaska. Cetology 44:1-19

Smedes F, Askland T (1999) Revisiting the development of the Bligh \& Dyer total lipid determination method. Mar Pollut Bull 38(3):193-201

Smith SJ, Iverson SJ, Bowen WD (1997) Fatty acid signatures and classification trees: new tools for investigating the foraging ecology of seals. Can J Fish Aquat Sci 54:1377-1386

Springer AM, Speckman SG (1997) A forage fish is what? Summary of the symposium. In: Forage fishes in marine ecosystems. Proc Int Symp Role of Forage Fishes in Marine Ecosystems. University of Alaska Sea Grant College Program, Report No. 97-01, p 773-805

Stevens J (1986) Applied multivariate statistics for the social

Editorial responsibility: Otto Kinne (Editor),

Oldendorf/Luhe, Germany sciences. Lawrence Erlbaum Associates, New Jersey

St. John MA, Lund T (1996) Lipid biomarkers: linking the utilization of frontal plankton biomass to enhanced condition of juvenile North Sea cod. Mar Ecol Prog Ser 131: $75-85$

Sturdevant MV (1996) Diet overlap of forage fish species. In: Duffy DC (ed) APEX: Alaska predator ecosystem experiment. Exxon Valdez Oil Spill Restoration Project annual report, No. 95163, Appendix C. Alaska Natural Heritage Program, University of Alaska, Anchorage

Vermeer K (1992) The diet of birds as tools for monitoring the biological environment In: Vermeer K, Butler RWH, Morgan $\mathrm{KH}$ (eds) The ecology, status and conservation of marine and shoreline birds on the west coast of Vancouver Island. Can Wildl Serv Occas Pap 75:41-50

Willette M, Sturdevant M, Jewett S (1997) Prey resource partitioning among several species of forgae fishes in Prince William Sound, Alaska. In: Forage fishes in marine ecosystems. Proc Int Symp Role of Forage Fishes in Marine Ecosystems. University of Alaska Sea Grant College Program, Report No. 97-01, p 11-29

Willette TM, Cooney RT, Hyer K (1999) Predator foraging mode shifts affecting mortality of juvenile fishes during the subarctic spring bloom. Can J Fish Aquat Sci 56: 364-376

Xu R, Hung SSO, German JB (1993) White sturgeon tissue fatty acid compositions are affected by dietary lipids. J Nutr 123:1685-1692

Submitted: January 11, 2002; Accepted: April 30, 2002 Proofs received from author(s): September 10, 2002 